\title{
Sistemas Tutores Inteligentes que Detectam as Emoções dos Estudantes: um Mapeamento Sistemático
}

\section{Intelligent Tutoring Systems that detect students' emotions: a systematic mapping review}

Helena M. Reis

Universidade de São Paulo

Instituto de Ciências Matemáticas e Computação

(ICMC)-São Carlos, Brasil

helenamcd@icmc.usp.br

Seiji Isotani

Universidade de São Paulo

Instituto de Ciências Matemáticas e Computação

(ICMC) - São Carlos, Brasil

sisotani@icmc.usp.br

\author{
Patrícia A. Jaques \\ Universidade do Vale do Rio dos Sinos \\ Programa de Pós-graduação em Computação \\ Aplicada - São Leopoldo, Brasil \\ pjaques@unisinos.br
}

\begin{abstract}
Resumo
As emoções vivenciadas pelos estudantes podem influenciar negativamente ou positivamente o aprendizado. Dessa forma, a detecção das emoções dos estudantes por softwares educacionais, como Sistemas Tutores Afetivos (STAs), permite a esses sistemas fornecer uma resposta mais adequada com objetivo de maximizar o aprendizado. Esse artigo apresenta um mapeamento sistemático que foi conduzido com o objetivo de investigar os mecanismos de deteç̧ão das emoções empregados por STAs. No total, foram analisados 462 estudos na área. Dentre estes estudos, 84 deles estavam relacionados com STAs e apenas 40 deles satisfizeram os critérios de inclusão e exclusão definidos neste trabalho. Como resultado, verificou-se que a maioria dos estudos usa a análise das expressões faciais como fonte principal de reconhecimento da emoção do estudante, seguido de sinais fisiológicos, linguística e dados comportamentais. Embora existam vários mecanismos para a detecção da emoção do estudante, ainda há carência de estudos que expliquem a relação entre emoções e aprendizado, bem como quais caminhos devem ser seguidos utilizando estas emoções com o propósito de tornar o ensino e a aprendizagem mais efetivos.
\end{abstract}

Palavras-Chave: Sistema Tutor Afetivo, Computação Afetiva, Emoções, Aprendizagem

\begin{abstract}
Emotions experienced by students can positively or negatively influence learning. Recognition of students' emotions during learning by educational software, such as Affective Tutor Systems (ATSs), allow these systems to provide a more adequate response to maximize learning. This paper presents a systematic mapping that was conducted to investigate the mechanisms of students' emotion detection that have been used by ATSs. In total, 462 studies in the area were analyzed. Among these, 84 studies were specifically related to ATSs and only 40 of them satisfied the inclusion and exclusion criteria defined in this work. As a result, we verified that most of the studies have used the analysis of facial expressions as the primary source of detection of the student's emotional state, followed by physiological, linguistic, and behavioral data. Although the findings suggest that there are several mechanisms for recognizing the student's emotional state, there is still a lack of studies that explain the relationship between emotions and learning, as well as which paths should be followed using these emotions to make teaching and learning more effective.
\end{abstract}

Keywords: Affective Tutoring System, Affective Computing, Emotions, Learning

Cite as: Reis, H. M., Jaques, P. A. \& Isotani, S. (2018). Intelligent Tutoring Systems that detect students' emotions: a systematic mapping review (Sistemas Tutores Inteligentes que Detectam as Emoções dos Estudantes: um Mapeamento Sistemático). Brazilian Journal of Computers in Education (Revista Brasileira de Informática na Educação - RBIE), 26(3), 76-107. DOI: 10.5753/RBIE.2018.26.03.76 


\section{Introdução}

Apesar da instrução individualizada de humanos-para-humanos ainda ser um dos mais efetivos meios de ensino, algumas características dos tutores humanos ainda são limitantes. Os professores nem sempre conseguem fornecer feedback imediato, pois raramente estão livres e prontos momentaneamente quando são solicitados. Além disso, os tutores humanos apresentam fadiga, mostrando um bom desempenho somente nas primeiras horas (D'Mello e Graesser, 2013).

Os sistemas tutores inteligentes (STIs) foram criados com o objetivo de fornecer assistência individualizada aos estudantes, simulando o comportamento de um tutor humano (Lane, 2006), quando este não está presente. Os STIs são sistemas computacionais educacionais que provêm instruções imediatas e customizadas aos estudantes. Essas instruções são geralmente realizadas sem intervenção humana (D'Mello e Graesser, 2013).

Apesar dos STIs serem quase tão efetivos quanto os tutores humanos (VanLehn, 2011), estes últimos possuem uma capacidade que falta à maioria dos STIs. Por exemplo, os tutores humanos podem perceber o estado afetivo do estudante em tempo real, e ajustar a estratégia pedagógica de acordo com essa percepção; característica essa que poucos STIs atuais possuem. Os tutores humanos conseguem regular suas estratégias de ensino baseadas no feedback não verbal (incluindo emocional) dado pelo estudante, que determina se ele está feliz, confuso, frustrado, surpreso, entediado ou contente com o material que o tutor está apresentando (Sarrafzadeh et al., 2011).

Overmyer (1999) verificou que a razão dos STIs serem geralmente menos efetivos do que tutores humanos deve-se ao fato da maioria desses sistemas não considerarem o estado afetivo do estudante, o que reduz drasticamente a sua habilidade de se adaptar às necessidades individuais do aprendiz. O mesmo ocorre em situações colaborativas de aprendizagem (Reis et al., 2015). Muitos estudos mostram que 35\% dos comentários feitos pelos tutores humanos possuem conteúdo emocional (Mao e Li, 2010), e estima-se que cerca de 93\% da comunicação afetiva acontece de maneira não verbal ou paralinguistica (Mehrabian, 1971), por meio das expressões faciais, gestos (correspondendo 55\% da emoção), ou entonações da voz (correspondendo a 38\% da emoção) (Stefanelli, 1993; Picard, 1998). Por exemplo, durante o processo de ensinoaprendizagem, o professor utiliza com frequência a linguagem não verbal para ensinar, mesmo que seja de forma inconsciente. $\mathrm{O}$ corpo do professor emite emoções, como sentimentos de serenidade, equilíbrio ou ansiedade, dependendo da situação vivida, podendo induzir efeitos positivos ou negativos aos estudantes (Silva e Silva, 2016). A comunicação entre o professor e o estudante deve contar com o bom desempenho e equilíbrio dos aspectos verbais e não-verbais (Carrasco, 2001). McNeill (1995) considera que os dois aspectos, verbal e não verbal, formam um todo que não pode ser dissociado ao se considerar os processos de comunicação no momento de ensino-aprendizagem (Barbosa et al., 2009). Desta forma, assim como os professores humanos, os tutores artificiais devem ser capazes de responder às emoções dos estudantes e, para isso, é necessário que sejam capazes de detectar as emoções destes estudantes.

Este artigo apresenta os resultados de um mapeamento sistemático ${ }^{1}$ (Petersen et al., 2008) com o objetivo de investigar os mecanismos de reconhecimento (i.e. detecção ou inferência de emoções) da emoção do estudante mais utilizados nos STAs. Esse estudo é uma extensão do artigo de Reis et al. (2017), sendo mais investigado profundamente a fundamentação teórica, as principais emoções detectadas nos STAs e quais tipos de STAs têm sido mais desenvolvidos.

Este artigo é organizado da seguinte forma: na Seção 2 são apresentados os principais conceitos relacionados a STAs e como as emoções afetam na aprendizagem. Na Seção 3, são

\footnotetext{
${ }^{1}$ É um método que envolve a busca por literatura a fim de verificar a natureza, a extensão e a quantidade de estudos publicados (chamados de estudos primários) na área de interesse (Borges et al., 2013).
} 
apresentados conceitos relacionados a Computação Afetiva e os tipos de detecção automática da emoção. A Seção 4 apresenta o método de pesquisa empregado. As Seções 5 e 6 mostra a análise e discussão dos resultados do mapeamento sistemático, respectivamente. As Seções 7 e 8 são apresentadas as ameaças à validade e a conclusão.

\section{Sistemas Tutores Afetivos}

Os Sistemas Tutores Inteligentes (STIs) são sistemas computacionais educacionais que têm o objetivo de promover instrução imediata e customizada aos estudantes a fim de proporcionar um ensino individualizado (Vos, 1995; VanLehn, 2006; Bernacki et al., 2014; Latham et al., 2014; Lin et al., 2014). Essas instruções são realizadas sem a intervenção humana, fazendo com o que o sistema simule o comportamento de um tutor humano e apresente dicas para auxiliar o estudante a desenvolver corretamente o problema ou identificar erros (Wenger, 1987; Azevedo et al., 1999; Curilem et al., 2007; Koedinger et al., 2009; Woolf, 2010; Adams et al., 2014).

A simulação do comportamento do tutor humano é feita por meio de técnicas de Inteligência Artificial, baseado em diversas abordagens, como a teoria de decisão e redes bayesianas, processamento de linguagem natural ou psicologia cognitiva (Bernacki et al., 2014). As características cognitivas dos estudantes são relevantes, e as instruções apresentadas a eles, seguem teorias pedagógicas (Azevedo et al., 1999).

Quando um STI considera também o estado afetivo para uma aprendizagem individualizada, é chamado de Sistema Tutor Afetivo (STA) (Ammar et al., 2010; Sarrafzadeh et al., 2011; Calvo e D'Mello, 2012). Os STAs são sistemas tutores inteligentes que monitoram os estados afetivos do estudante a fim de igualmente prover feedback adaptado às emoções do aprendiz durante o ensino (Mao e Li, 2010; Calvo e D'Mello, 2012; D'Mello e Graesser, 2013). O STA é destinado a se adaptar à emoção de forma efetiva, imitando um cenário humano real (Sarrafzadeh et al., 2004) com o objetivo de estimular os estudantes a aprenderem.

Um STA pode responder apropriadamente às emoções negativas do estudante e proporcionar experiência de aprendizado desafiante. Kappor et al. (2007) sugere que o reconhecimento do estado afetivo do estudante, por meio do computador, pode ser um pouco mais preciso em comparação com outras técnicas (e.g. observação ou autorrelato). Isso deve-se ao fato de que estudantes podem frequentemente esconder sua emoção, por exemplo, ao se transparecer bem, quando na verdade não estão (Schneider e Josephs, 1991). O autorrelato sobre a emoção, ao final das tarefas realizadas pelos estudantes, não é confiável. Atualmente, classificar a emoção do estudante se torna uma tarefa onerosa e burocrática, pois requer vários observadores para validar a emoção de cada indivíduo.

\subsection{Emoções e Aprendizagem}

De acordo com Scherer (2000), emoções são definidas como respostas às situações que são percebidas como relevantes para avaliação de um evento interno ou externo de maior importância. Raiva, tristeza, alegria, medo, vergonha, orgulho e desespero são exemplos de emoções. Uma recente revisão das emoções em sistemas computacionais educacionais (D'Mello e Graesser, 2013) revelou que há um número qualitativo diferente de emoções que podem ser medidas, variando das teorias universais de Ekman e Friesen, emoções básicas (e.g. raiva, tristeza, felicidade) a teorias de aprendizagem de emoções, como Pekrun (2006) e emoções centrais do estudante (e.g. orgulho, ansiedade, confusão, tédio e divertimento) de Graesser e D’Mello (2014). As emoções também podem ser divididas em primárias, secundárias e terciárias (Bicho e Pereira, 2007). As emoções primárias podem ser sentidas por qualquer ser humano independentemente da 
cultura. As emoções secundárias podem ser atividades e expressadas dependendo de cada cultura. E, por fim, as emoções terciárias são induzidas por elementos internos em conjunto com processos físicos ou interações com o meio, por exemplo, esforço físico prolongado.

As emoções possuem um papel importante na aprendizagem. Para Piaget (1989), sem afetividade o sujeito não teria interesse para desvendar problemas ou fazer descobertas. A inexistência de perguntas, por falta de interesse ou motivação, podem representar um obstáculo para o desenvolvimento da inteligência. De acordo com Piaget (1989), é incontestável que as emoções aceleram ou perturbam a aprendizagem, e que não existe aprendizagem sem afetividade e vice-versa. Pekrun (2011) sugere que as emoções negativas possam impedir a aprendizagem, ativando a ansiedade e a raiva, tédio e a falta de esperança. As emoções positivas podem promover o aprendizado, ativando emoções como engajamento, curiosidade e orgulho (Fredrickson, 1998). Por outro lado, a confusão tem sido associada ao aprendizado profundo (e.g. melhor entendimento e retenção mais significativa do material), dependendo das circunstâncias (Calvo e D'Mello, 2012). Entretanto, a confusão pode liberar emoções negativas ao aprendizado, como frustrações, quando os estudantes não conseguem realizar as atividades e nem entender as instruções (D'Mello et al., 2014). Neutralidade (emoções neutras) facilita a aprendizagem, pois envolve o estudante em um estado psicológico onde não é distraído por emoções negativas, além de ter disposição para o aprendizado (Jaques et al., 2014; Harley et al., 2016).

Alguns estudos reportaram que as emoções dos estudantes podem ser ativadas e desativadas por eventos negativos e positivos, sendo classificadas em quatro categorias (Pekrun, 2006): (a) emoções positivas (e.g. divertimento, alegria, esperança e felicidade) são acionadas por eventos que trazem benefícios, e introduzem uma forte motivação de aprendizado nos estudantes para continuarem aprendendo; (b) desativação de emoções positivas (e.g. alívio ou relaxamento) são tipicamente estimuladas depois de acabar um evento negativo, causando aos estudantes um relaxamento temporário durante o processo de aprendizagem. No entanto, tais emoções são favoráveis depois de um certo tempo decorrido o incidente; (c) ativação de emoções negativas incluem ansiedade e vergonha; estudantes com tais emoções são caracterizados por um nível alto de controle, que os motiva a resolver problemas encontrados durante o aprendizado ou dedicar um tempo adicional para evitar o fracasso; (d) desativação das emoções negativas (e.g. tédio e falta de esperança) é gerada quando as atividades de aprendizado ou resultados demandam além das capacidades ou tolerância dos estudantes, ou quando esses não estão dispostos a superar os desafios durante o aprendizado. Além disso, estudantes com desativação das emoções negativas são caracterizados por falta de controle subjetivo.

Embora se observe um interesse crescente pelos pesquisadores da área de Computação Afetiva nas pesquisas que relacionam a emoção e a aprendizagem (Calvo e D'Mello, 2012), muitas questões continuam sem respostas. Por exemplo, investigações mais profundas são necessárias para saber quais emoções são relevantes para a aprendizagem, bem como quais caminhos devemos seguir utilizando essas emoções para fazer o ensino e a aprendizagem mais efetivos. Do ponto de vista de modelos e métodos para testar a emoção, há um grande avanço nas pesquisas; mas do ponto de vista de explicar a relação entre emoções e aprendizado, há uma carência de investigações (Hernández et al., 2015).

\section{Computação Afetiva e Detecção Automática da Emoção}

A computação afetiva é uma área de pesquisa que estuda e investiga o desenvolvimento de sistemas que dão aos computadores a habilidade de reconhecer, interpretar, processar, expressar e simular emoções humanas (Picard, 1997). Além disso, os sistemas devem interpretar a emoção dos usuários e adaptar o seu comportamento a eles, dando uma resposta adequada para essas emoções (Tao e Tieniu, 2005). 
Os trabalhos em computação afetiva podem ser classificados em duas principais subáreas, segundo Jaques e Vicari (2007), conforme Figura 1. A primeira (Emoções na Interação HumanoComputador) envolve os sistemas que detectam (reconhecem) e respondem às emoções dos usuários com o objetivo de produzir mais engajamento e interações personalizadas aos seus estados afetivos (Calvo e D'Mello, 2012). Já a segunda subárea (Síntese de Emoções) trata dos sistemas inteligentes que modelam emoções, o que possibilita a tomada de decisão de forma mais eficaz, e semelhante à tomada de decisão humana, em comparação com os sistemas puramente racionais (Calvo e D'Mello, 2012). Um exemplo são os agentes pedagógicos (com arquitetura interna de emoções) que auxiliam os estudantes no processo de ensino-aprendizagem, fornecendo dicas e feedback (Harley et al., 2016).

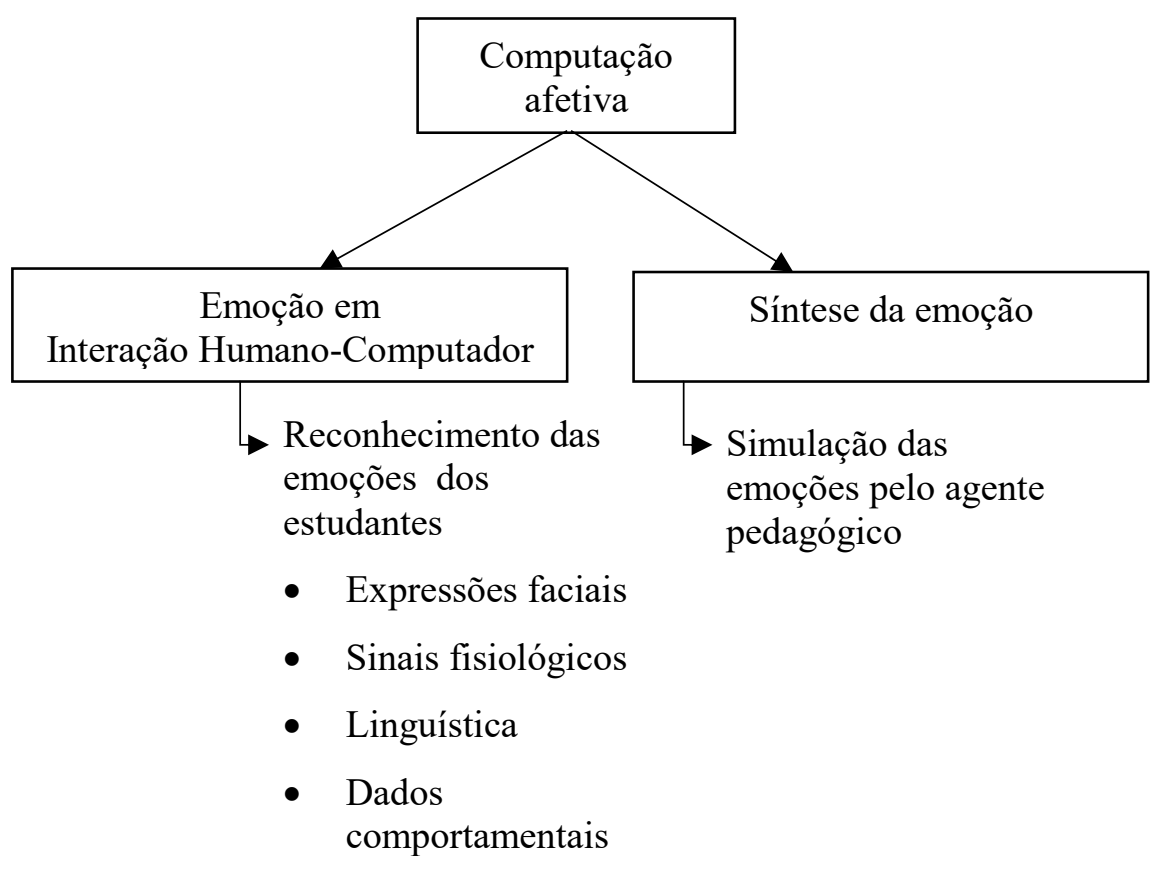

Figura 1: Classificações dos trabalhos na área de Computação Afetiva. Adaptado de Jaques e Vicari (2007).

Os Sistemas Tutores Afetivos se encontram na primeira sub-área: Emoções na Interação Humano-Computador. Esses sistemas devem ter mecanismos para detectar as emoções dos estudantes para que assim possam responder a esses estados afetivos de forma personalizada. Jaques e Vicari (2007) classifica o reconhecimento da emoção do usuário em quatro grupos centrais: (i) expressõoes faciais, (ii) sinais fisiológicos (batimentos cardíacos, tensão muscular, condutividade da pele e respiração), (iii) dados comportamentais, que são ações e escolhas do usuário na interface do sistema (e.g. opções de escolha, velocidade, de digitação), e (iv) linguística (voz e semântica). As formas de reconhecimento usadas para essas fontes de dados são descritas em mais detalhes nessa seção.

\subsubsection{Expressões faciais}

Apesar dos seres humanos terem a capacidade de se expressar por meio da voz, a importância das expressões faciais, durante a interação social, também deve ser considerada. Essas expressões podem ser medidas por espontaneidade ou voluntariedade (Schyns et al., 2009) e são um dos meios mais importantes de comunicação não verbal. As expressões faciais podem ser diversas, como movimentos da cabeça ou dos olhos, sobrancelhas erguidas, franzir a testa (Nkambou, 2006; Ammar et al., 2010), direção do olhar ou alteração do diâmetro da pupila (D'Mello et al., 2012; 27; Jaques et al., 2014). 
Diversos estudos (Rosemberg e Elkman, 1994; Ruch, 1995; Sarrafzadeh et al., 2011) têm indicado que há alta relação entre as expressões faciais espontâneas (ou inconscientes) e as emoções. A detecção das emoções por meio das expressões faciais tem sido realizada em diversas áreas, dentre elas, em ambientes educacionais (Tian et al., 2005; Khan et al., 2011; Khan et al., 2013; Cabada et al., 2014; Harley et al., 2016). A maioria desses ambientes visam reconhecer um pequeno conjunto de expressões faciais emocionais relacionadas às seis emoções básicas ou a um subconjunto delas, e essas acontecem poucos nas situações de aprendizagem.

De acordo com Babad (2005), o processo de ensino-aprendizagem dentro do ambiente educacional é impactado pela interação não verbal. Por meio das expressões faciais dos estudantes, os professores conseguem inferir a emoção do indivíduo no momento da instrução. Dependendo do resultado da avaliação, o professor pode reavaliar a forma da sua explicação ou conteúdo que está sendo ensinado. Por outro lado, os estudantes conseguem sentir o feedback de suas ações por meio das expressões faciais do seu professor, que podem variar entre diversas emoções, como medo, dúvida, reprovação ou orgulho (Matsumoto e Hwang, 2011).

Um cenário real seria o estudo de Silva e Silva (2016), que investigou como as expressões faciais afetam no ensino da Enfermagem. Os resultados sugerem que expressões como o sorriso podem gerar efeitos positivos, como positividade em relação ao que o estudante falava, encorajamento, apoio e incentivo, mas também efeitos negativos como desdenho, insatisfação e deboche quando os professores escutavam e analisavam o processamento dos conteúdos curriculares pelos estudantes.

Dentro dos ambientes educacionais computacionais, as emoções dos estudantes podem ser medidas por meio de dispositivos tecnológicos que extraem as características faciais dos estudantes durante o ensino (D'Mello et al., 2010). Há duas abordagens principais que são utilizadas na extração de características faciais: métodos baseados em características geométricas e métodos baseados em aparência. Os métodos baseados em características geométricas da face utilizam as posições e as distâncias entre pontos faciais, a velocidade de separação destes, dentre outras. As caraterísticas faciais normalmente utilizadas são o formato da boca, dos olhos, sobrancelhas e nariz. Nelas são analisados os cantos dos olhos, da face e da boca (Cabada et al., 2015). No contexto das características verificam-se as mudanças físicas, como o surgimento de rugas ou marcas de expressão, que podem ser observadas pela textura da pele. Nesse método, a face pode ter suas características representadas por mapas de intensidade, de cores, pela aplicação de filtros, dentre outros.

Alguns estudos (D'Mello et al., 2010; Jaques et al., 2014) vêm investigando o uso de rastreadores oculares para extrair as características faciais dos estudantes para inferir suas emoções. O estudo de D'Mello et al. (2012) investigou o uso de rastreadores oculares para rastrear períodos de falta de engajamento dos estudantes durante o ensino. Ao detectar tédio, por parte dos estudantes, era lançada uma nova tentativa de reorientar a atenção dos estudantes por meio de um agente pedagógico animado, melhorando a qualidade do ensino. Por exemplo, caso o estudante não esteja olhando para tela ou um componente da interface (i.e. imagem, textbox ou vídeo) por um longo período de tempo, o sistema considera que o estudante apresenta desmotivação e falta de atenção (D'Mello et al., 2010; Smilek et al., 2010). Dessa forma, o rastreamento ocular pode ser usado para detectar a alteração da atenção ou a presença de tédio do estudante para melhorar as saídas do sistema. Contudo, essa técnica de reconhecimento emocional pode gerar um resultado incorreto caso o estudante feche os olhos para dirigir a atenção a algum componente de som ou realizar um pensamento introspectivo.

Apesar das expressões faciais das emoções serem universais, a cultura influencia na forma como as pessoas expressam facilmente as suas emoções e como as gerem (Padrón-Rivera et al., 2016). Os estudantes japoneses mostram maior inibição para expressar certas emoções quando estão em presença de uma figura de autoridade, diferentemente dos estudantes americanos 
(Ekman, 2003). Segundo Vail et al. (2016), além das diferenças culturais, há também diferenças de expressões faciais entre homens e mulheres. $\mathrm{O}$ estudo sugere que as mulheres apresentam maiores expressões faciais quando identificam a expressão facial dos outros, além de conseguirem identificar mais precisamente a emoção do outro quando estão conversando.

\subsubsection{Sinais fisiológicos}

Sensores fisiológicos e emocionais estão surgindo como uma forma tecnológica para o reconhecimento da emoção do estudante durante o ensino (Allanson e Fairclough, 2004; Lin et al., 2016; Picard, 1997). Esses sensores são capazes de detectar desde mudanças físicas externas do estudante (e.g. mudança de postura), até mudanças internas (e.g. frequência cardíaca ou condução elétrica da pele) e possuem a vantagem de poderem ser aplicados em diversos domínios e conteúdo. Entretanto, são difíceis de serem utilizados em situações da vida real, por serem considerados invasivos e pelo alto custo de alguns sensores (Shanabrook et al., 2016).

O uso desses sensores é possível devido a diversos órgãos do corpo humano, tais como o coração, o cérebro, os músculos ou os olhos, emitirem sinais elétricos (Feldman e Goldwasser, 2004). Por exemplo, o coração produz um sinal registrado pelo eletrocardiograma (ECG), o cérebro produz um sinal que é registrado pelo eletroencefalograma (EEG) e a atividade muscular, como a contração ou relaxamento, é registrada pelo eletromiograma (EMG).

Alguns desses sinais podem ser decompostos em outros, como o ECG, que pode emitir medidas e variações de frequência cardíaca. Essas medidas, em conjunto com medidas de outros sensores, podem ser usadas para detectar as emoções dos estudantes (Harley et al., 2013). Levenson et al. (1992) sugere que as emoções de medo, raiva e tristeza produzem uma maior escala de aceleração da frequência cardíaca do que felicidade, aversão e surpresa, isso porque a frequência cardíaca é um indicador da valência da emoção.

Os sensores fisiológicos podem ser usados em conjunto para tentar aferir com maior precisão as emoções dos estudantes. O estudo de Alzoubi et al. (2012) utilizou os sensores de ECG, EMG e GSR (reposta galvânica da pele) para reconhecer as emoções de um estudante durante o uso do AutoTutor no domínio de Sistemas Operacionais, Internet e Hardware. Nesse estudo, os autores investigaram duas abordagens que têm sido adotadas para o desenvolvimento de detectores de emoções baseados na psicologia: modelo independente do usuário e modelo dependente do usuário. No modelo dependente do usuário, os modelos de classificação são construídos a partir de um ou mais registros gravados de um único indivíduo e é calibrado de acordo com padrão da emoção e psicológico dele. Por outro lado, o modelo independente de usuários tem como objetivo construir modelos a partir de dados de vários indivíduos e é esperado que generalize novos indivíduos. Os resultados demonstram que os desenvolvimentos a partir de modelos independentes de usuários não são precisos, possivelmente devido às diferenças individuais nas respostas durante o uso do AutoTutor. No entanto, os modelos dependentes dos usuários alcançaram precisão moderada. $\mathrm{O}$ uso de somente um sensor fisiológico não é confiável ou suficiente para determinar a emoção do estudante. Os sensores devem ser utilizados em conjunto ou ainda com câmeras, para o reconhecimento facial, sensores de postura e de voz (Sarrafzadeh et al., 2011; Baker et al., 2014).

Os sensores de postura são instalados nas cadeiras dos estudantes para verificar se ocorre uma pressão elevada no assento. Uma mudança de movimentação, como por exemplo, inclinar o corpo para a frente para visualizar melhor a interface do sistema, pode ser detectado por este sensor e representar que o estudante está atento às instruções apresentadas. Por outro lado, um aumento de pressão sobre o descanso das costas da cadeira sugere que o estudante esteja inclinado para trás, podendo sugerir baixa atenção. Ao analisar a alternância entre a pressão do assento e o descanso das costas, é possível sugerir o interesse (interessado ou desinteressado) do estudante, ao utilizar o sistema, sobre o conteúdo ensinado (Vail et al., 2016). 
Outro sensor fisiológico utilizado para medir a emoção do estudante é o GSR. Esse sensor é considerado sensível às mudanças, tendendo a aumentar o sinal com algum estímulo emocional, como música ou violência, e diminui quando as emoções são menos intensas (Lin et al., 2016). Entretanto, o estudo de Harley et al. (2015) investigou três métodos de medições emocionais, como expressão facial, autorrelato de emoções e o uso do GSR. Os resultados demonstraram que as expressões faciais e o autorrelato dos estudantes possuíam alta concordância entre si, porém havia baixa concordância destes com o sensor GSR, demonstrando que nem sempre existe um relacionamento exato entre as emoções e os componentes de resposta emocional. O sensor fisiológico pode não apresentar exatidão no reconhecimento emocional do estudante, gerando falsos positivos.

Outros estudos (Kapoor et al., 2007; Sarrafzadeh et al., 201; Lin et al., 2016; Harley et al., 2016; Vail et al., 2016) investigaram o uso da eletromiografia (EMG) para detectar as emoções faciais dos estudantes, por meio da medição da contração dos músculos da face, mesmo quando este movimento não é visível. A atividade fisiológica é observada e acompanhada de diversas expressões faciais espontâneas (Ekman e Davidson, 1993), que também demonstra ser uma ligação entre emoção e expressões faciais. $O$ estudo sugere que as expressões faciais acompanhadas de atividades físiológicas são relacionadas com as emoções (Sarrafzadeh et al., 2011; Lin et al., 2016).

E, por fim, o eletroencefalograma (ECG) é uma variável fisiológica de atividade elétrica envolvendo o couro cabeludo e tem sido correlacionado com atenção, memória e percepção. Esses elementos afetam as emoções dos estudantes, como engajamento e motivação (Norman, 1981). Apesar dos altos custos que esses sensores possuem para a pesquisa e por não possuírem custobenefício para aplicação generalizada, Golderberg et al. (2012) investigou a eficácia do uso de sensores de baixo custo para reconhecer os estados emocionais dos estudantes. Os resultados demonstraram que esses sensores são confiáveis para a utilização neste contexto.

\subsubsection{Dados comportamentais}

O comportamento é tudo o que pode ser observado e tudo o que responde à mudança em contingências de reforço (Todorov, 2012). Dentro de um sistema computacional, esse comportamento pode ser caraterizado pelas escolhas e ações do usuário, chamados de dados comportamentais. Esses dados podem ser armazenados para uma posterior análise, com o objetivo de conhecer o usuário, adaptar o sistema de acordo com suas características, entre outros (Jaques e Vicari, 2007).

Uma forma de coletar dados comportamentais sobre como os usuários utilizam o sistema seria através de registros feitos durante o uso. Isso pode ser feito por meio de logs, que armazenam em um arquivo as ações executadas em um sistema, por meio da gravação da interação do usuário com o sistema, ou da gravação em vídeo da experiência do usuário. As diferentes formas de registro armazenam aspectos distintos do uso. Normalmente, registros de uso geram uma grande quantidade de informação e a análise desses dados pode ser bastante custosa para avaliadores (Muñoz et al., 2011).

No contexto da educação, os dados comportamentais podem apoiar a análise das emoções durante o processo de ensino-aprendizagem. Segundo Jaques e Vicari (2007), as emoções podem ser inferidas a partir dos dados comportamentais do estudante, como ações do estudante na interface do ambiente educacional. Os autores citam alguns exemplos de ações: tempo para realizar um exercício, sucesso ou falha na realização de tarefas, pedido de ajuda ou negação de uma ajuda, velocidade da digitação do estudante, entre outros (Lagud e Rodrigo, 2010; Doddannara et al., 2013). O estudo de Shanabrook et al. (2016) cita a extração dos dados comportamentais a partir da velocidade dos movimentos dos dedos e da pressão que o estudante realiza ao tocar uma tela sensível ao toque. 
Outro estudo (Allen et al., 2016) investigou os dados comportamentais durante a digitação de um estudante. Os resultados sugerem que o estudante apresenta tédio quando digita palavras mais curtas, textos menores e tem maior frequência de pausas longas entre a digitação de uma palavra e outra. Em contrapartida, estudantes engajados tem menores pausas entre digitação de uma palavra e outra, e sua escrita é mais fluída.

Por meio dos dados comportamentais do estudante, o sistema deve realizar análise e processamento sobre a situação e tentar inferir as emoções do usuário usando um modelo psicológico de emoções. Normalmente, esses sistemas utilizam um modelo psicológico cognitivo de emoções (e.g. modelo OCC ou Control-Value Theory) para inferir a emoção do estudante (Jaques e Vicari, 2007). O modelo psicológico utiliza as informações fornecidas e assim constrói uma interpretação de uma situação do ponto de vista do usuário e raciocina sobre qual emoção essa interpretação leva (Jaques e Vicari, 2007). Por exemplo, para o modelo OCC inferir a emoção feliz, o sistema raciocina sobre o aspecto desejável de um evento em relação aos objetivos do estudante (Jaques e Vicari, 2007; Muñoz et al., 2011). Se o estudante tem o objetivo de agradar aos pais, obter uma nota boa em um exercício se torna um evento desejável (situação), já que ele promove seus objetivos e, dessa maneira, dispara emoções positivas como felicidade. Para Muñoz et al. (2011), o modelo OCC consegue atingir 70\% de precisão, que pode ser aumentada em 10\% quando o modelo é combinado com dados coletados por meio dos sensores fisiológicos.

O modelo OCC pode ser combinado com a rede Bayesiana dinâmica para relacionar, probabilisticamente, informações sobre o estudante, como a personalidade, os objetivos, e eventos de interação com a emoção dele, visando melhorar a acurácia do resultado (Conati, 2002; Conati e Maclaren, 2009; Hernández et al., 2015). Outra informação utilizada seria a intensidade da emoção. De acordo com o modelo OCC, as emoções surgem da avaliação de eventos, pessoas ou coisas. Alegria e tristeza são emoções opostas que surgem a partir da avaliação dos eventos, assim como satisfação e frustração. Gratidão e raiva surgem da avaliação das ações de outro agente, bem como orgulho e vergonha são ações de si mesmo (Jaques e Vicari, 2007).

Apesar da possibilidade de inferir as emoções do estudante por meio dos dados comportamentais, esta é uma tarefa difícil e não fornece exatidão. Porém, essa dificuldade da acurácia pode ser minimizada com o uso de sensores fisiológicos que detectam expressões corporais e podem ser usados em conjunto com as informações inferidas pelos dados comportamentais do estudante (analisadas de acordo com um modelo cognitivo de emoções) para determinar a intensidade das emoções com maior precisão. Estes sensores fisiológicos são úteis para identificar quando o estudante sente uma emoção, a valência e a intensidade delas (Jaques e Vicari, 2007).

\subsubsection{Linguística}

A voz é uma das principais formas de comunicação e uma das mais ricas fontes de informação emocional (Williams e Stevens, 1972; Smith et al., 1975). A comunicação ocorre não somente pelo significado da palavra pronunciada, mas também por meio de expressões vocais não verbais, como a entonação ou volume (modificações de voz durante a fala); e por meio de vocalizações não faladas, como a respiração, gestos corporais, gestos faciais, gritos e soluços. Dependendo da entonação da voz, uma mesma palavra pode ser expressa de diferentes maneiras e provocar diferentes emoções (Thurman e Welch, 2000). Dessa forma, um elogio pode parecer uma crítica ou um pedido pode soar como uma ordem. Portanto, a forma como o ser humano lida com suas emoções se reflete na sua voz.

Diante desse contexto, os estudos de Kyrillos et al. (2003) e Santos e Mortimer (2001) investigaram a percepção da emoção de um diálogo apenas pelas variações da voz, da fala ou da expressão corporal, independentemente se o receptor conhece o idioma do locutor. O resultado do estudo demonstrou a importância de relacionar a linguagem, expressividade verbal e não-verbal 
e as emoções em sala de aula. Segundo Fabron (2006), a expressividade comanda a interação entre professor e estudante, podendo facilitar a construção do conhecimento e até mesmo garantir a atenção dos estudantes. Estes, por sua vez, podem identificar as emoções dos professores ao realizarem as atividades, e os professores podem identificar as frustrações, engajamento e motivações dos estudantes por meio das emoções reconhecidas pela comunicação não-verbal.

Apesar de parecer que os humanos podem perceber e processar diferenças nas propriedades da voz e utilizar essas informações para identificar a emoção do locutor, a tarefa de reconhecimento emocional pela voz é complexa e, muitas vezes, exige outros parâmetros como o conhecimento do contexto, do significado semântico da mensagem e da expressão facial da pessoa. Para tanto, nem sempre os humanos conseguem realizar esta análise perfeitamente. Algumas dessas evidências constam em estudos (Sharma e Kim, 2002; Razak et al., 2005; Tóth et al., 2008) que demostram que a habilidade humana, para reconhecer emoções somente pela voz, está entre $60 \%$ a $80 \%$. No contexto educacional, a falta de acurácia no reconhecimento emocional do estudante pelo professor pode afetar nas atividades em sala de aula. O professor pode não conseguir detectar que aquele estudante está apresentando uma emoção negativa, fazendo com que o processo de ensino aprendizagem fique comprometido.

Para reduzir percepções erradas de emoções por meio da voz, diversos sistemas automáticos já foram propostos ao longo dos anos, surgindo casos registrados onde a acurácia foi satisfatória, equiparando-se à humana, como (Hyun et al., 2005) com 67, 92\%; boa, superando a acurácia humana, como (Iliou e Anagnostopoulos, 2009) com 83,17\%; e excelente, beirando $100 \%$ de acertos, como (Lin et al., 2014) com 99,5\%. Vale ressaltar que o desempenho dos reconhecedores automáticos fica limitado às bases de dados utilizadas, ao número de emoções reconhecidas, ao número de locutores e às condições de independência de gênero e de locutor estabelecidas nos testes.

Algumas destas técnicas para o reconhecimento de emoções por meio da voz são aplicadas aos softwares educacionais, dentre eles os STIs. De todo modo, a maioria destes softwares não considera as expressões vocais não verbais, como a entonação da palavra falada, frequência ou o volume para determinar a emoção do estudante. A técnica mais utilizada nesses sistemas é a transformação das palavras pronunciadas em textos, fazendo a análise somente da prosódia, ou seja, do conteúdo das palavras, como apresentado nos estudos de Rajkumar e Ramalinga (2015) e Mao e Li (2010).

A mesma técnica é investigada no estudo de Calvo e D'Mello (2012), que utiliza o recurso da análise da semântica para detectar emoções dos estudantes por meio de um diálogo textual em linguagem natural. $\mathrm{O}$ objetivo do estudo era utilizar um agente pedagógico que lida com as emoções negativas dos estudantes, a fim de regulá-las. Os resultados demonstraram que os STIs que utilizaram esse recurso aumentaram o desempenho dos estudantes de baixo conhecimento no processo de ensino-aprendizagem e, no entanto, foram menos efetivos entre os estudantes que possuíam maiores conhecimentos.

Esse mesmo resultado foi alcançado por Lin et al. (2014), Jimenez et al. (2016) e Baker et al., (2010), e sugere que técnicas de reconhecimento da linguagem natural que tenham sido integradas em sofisticados STIs podem ajudar os estudantes a construírem seus próprios conhecimentos por meio da discussão, conseguindo contornar as emoções negativas dos estudantes durante o processo de aprendizagem.

O uso da análise da voz e semântica para o reconhecimento dos estados emocionais dos estudantes pode ser menos invasivo que os sensores fisiológicos, proporcionando ao estudante maior conforto, pois alguns deles se sentem incomodados quando percebem que estão sendo observados (Lin et al., 2015). 


\section{Método}

O método utilizado neste artigo foi o mapeamento sistemático. $\mathrm{O}$ foco deste estudo é limitado aos STAs que possuem algum mecanismo de reconhecimento da emoção do estudante. Mais especificamente, esse estudo objetiva investigar os trabalhos que desenvolveram e/ou empregaram mecanismos de reconhecimento, excluindo aqueles que apenas sugerem o seu uso, sem empregá-los empiricamente.

Este estudo baseou-se nas diretrizes propostas por Petersen et al. (2008) e foi conduzido de acordo com os cinco passos a seguir: (i) definição de questões de pesquisa, (ii) realização da pesquisa de estudos primários relevantes, (iii) triagem dos documentos, (iv) keywording dos resumos, e (v) extração de dados e mapeamento. primários.

Abaixo têm-se a descrição de forma detalhada do processo de busca e seleção dos estudos

\subsection{Definição de questões de pesquisa}

As questões de pesquisa definidas neste estudo são as seguintes:

QP1: Quais são as principais fontes de dados (por exemplo, voz, expressões faciais, log de ações, etc) de detecção das emoções dos estudantes em STAs?

QP2: Quais são as principais emoções detectadas nos STAs?

QP3: Quais são os principais tipos de STAs desenvolvidos?

\subsection{Condução da pesquisa}

A busca por estudos primários foi realizada nas bibliotecas digitais internacionais utilizando as palavras "emotion", "affective" e "sentiment", além das palavras "Intelligent Tutoring Systems", "Affective Tutoring Systems", "ITS" e "ATS". Também foi executada uma pesquisa nas bibliotecas digitais brasileiras com as palavras "emoção", "afeto", "afetividade", "sentimento" e "personalidade", além das palavras "sistemas tutores inteligentes" ou Sistemas Tutores Afetivos. A primeira parte da string é relacionada ao estado afetivo, como emoção, afetividade e sentimento, enquanto a segunda parte sobre os estudos relacionados sobre Sistemas Tutores Inteligentes ou Sistemas Tutores Afetivos. Nenhum campo em particular (filtro) foi usado para limitar a pesquisa, como a data de publicação.

É importante salientar que os termos em inglês foram usados nas buscas nas bibliotecas digitais internacionais e os termos em português apenas nas buscas nas bibliotecas nacionais. Embora possam haver casos de artigos em português publicados em bibliotecas internacionais ou artigos em inglês publicados nas bases nacionais, esses geralmente apresentam um resumo e título na outra língua. Dessa forma, acredita-se ser desnecessária a busca dupla (usando termos em português e inglês) em ambas as bases.

Após a construção da string de pesquisa, a busca foi conduzida nas principais bibliotecas digitais internacionais, todas consideradas propensas a retornar estudos relevantes no campo da Ciência da Computação, Educação e Pesquisa Educacional (Douglas-Cowie et al., 2007). As bibliotecas digitais utilizadas foram as seguintes: ACM Digital Library, Scopus, Web of Knowledge, Elsevier (via Science Direct) e IEEExplore. 
Houve uma busca manual nas bibliotecas digitais em português: na revista Informática na educação teoria e prática ${ }^{1}$, Revista Novas Tecnologias na Educação ${ }^{2}$, Revista Brasileira de Informática na Educação ${ }^{3}$ e artigos dos eventos do CBIE $^{4}$. A busca foi realizada entre 05/08/2016 e 10/08/2016.

\subsection{Critérios de seleção}

Os estudos selecionados foram analisados a fim de que fossem identificados aqueles relevantes ao contexto da pesquisa. Esta análise consistiu na aplicação de critérios de inclusão e exclusão previamente definidos. Os critérios de inclusão elaborados foram os seguintes:

- Se múltiplos artigos investigarem o mesmo software ou a mesma pesquisa, apenas o mais recente deve ser incluído;

- Se houver várias versões do mesmo artigo (e.g. artigo resumido ou completo), o mais completo deve ser incluído e;

- Artigos que abordam mecanismos para o reconhecimento dos estados emocionais em STAs.

Critérios de exclusão são importantes, pois permitem uma maior precisão na eliminação de estudos considerados não relevantes ao contexto da pesquisa em andamento. Por essa razão, durante a análise dos estudos retornados, todos aqueles que se enquadraram em ao menos um dos critérios de exclusão abaixo foram descartados.

- Artigos que não abordam mecanismos para o reconhecimento dos estados emocionais em STAs e;

- Relatórios técnicos, documentos que estão disponíveis em forma de artigos resumidos ou apresentação/slides e estudos secundários (revisões e mapeamento sistemático da literatura devem ser excluídos).

Inicialmente, 628 estudos primários foram retornados. Entretanto, 128 artigos eram duplicados, resultando 499 artigos candidatos. Destes, 38 artigos eram abertura de congressos e convites para participação de palestrantes em eventos, resultando 462 artigos candidatos. Após aplicar os critérios de inclusão e exclusão no título, resumo e palavras-chaves, foram obtidos 84 artigos candidatos. Por fim, depois de realizar a leitura na introdução e conclusão, o conjunto final selecionado foi de 35 artigos. Muitos artigos excluídos apenas mencionam a possibilidade de utilizar algumas técnicas de reconhecimento da emoção do estudante dentro de um STI, no entanto não abordam empiricamente o uso deste.

Nas bibliotecas nacionais, inicialmente, 44 estudos primários foram retornados. Destes, um estudo primário era uma versão resumida. Após aplicar os critérios de inclusão e exclusão no título, resumo e palavras-chaves, foram obtidos seis artigos candidatos. Por fim, depois de realizar a leitura na introdução e conclusão, o conjunto final selecionado foi de cinco artigos. No total do mapeamento, foram selecionados 40 artigos. A lista completa desses artigos pode ser obtida no Apêndice A.

Os artigos não selecionados nas bibliotecas digitais nacionais não passaram nos critérios de inclusão, pois apenas trataram de assuntos diversificados, não abordando STAs. Estes assuntos abrangeram o estudo do afeto na formação de grupos, formação de grupos considerando traços de personalidade, design afetivo, afetividade dentro de sistemas como Ambiente Virtual de Ensino e Aprendizagem (AVEAs), sendo estes assuntos investigados no estudo de Moraes et al. (2017).

\footnotetext{
${ }^{1} \mathrm{http}: / /$ seer.ufrgs.br/InfEducTeoriaPratica

${ }^{2}$ http:// http://seer.ufrgs.br/renote

${ }^{3} \mathrm{http}: / /$ www.br-ie.org/pub/index.php/rbie

${ }^{4}$ http://br-ie.org/pub/index.php/
} 


\section{Análise dos resultados}

Após a busca e seleção dos estudos primários, os artigos foram categorizados. Nesta etapa, verificou-se que as categorias eram similares à classificação proposta por Jaques e Vicari (2007), sendo eles expressões faciais, sinais fisiológicos, dados comportamentais e linguística (detalhados na seção 2.4).

Respondendo a QP1 (Quais são as principais fontes de dados (por exemplo, voz, expressões faciais, log de ações, etc) de detecção das emoções dos estudantes em STAs?), é possível observar na Figura 2 que a maioria dos estudos primários apresentam reconhecimento da emoção do estudante por meio das expressões faciais, com 21 estudos (46\%). Por outro lado, dentre os estudos primários selecionados, o reconhecimento da emoção por meio da linguística e dados comportamentais são os menos investigados: somente oito estudos primários, que contabilizam somente $17 \%$ dos estudos selecionados.

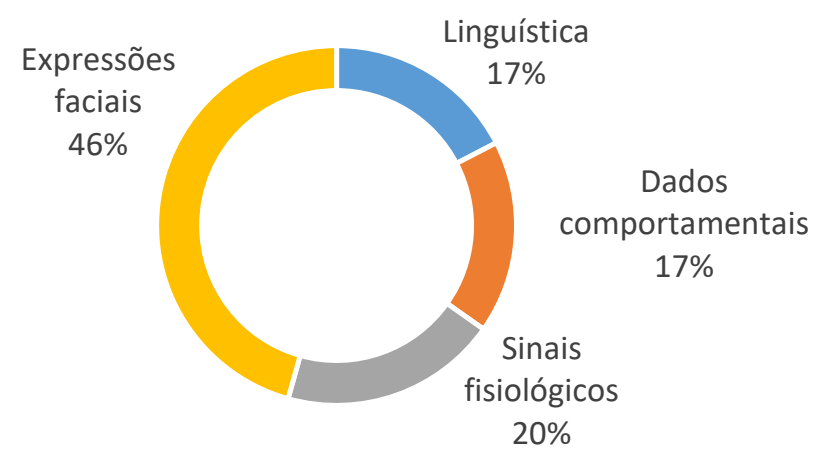

\begin{tabular}{|c|c|c|}
\hline Categoria & Frequência & Porcentagem (\%) \\
\hline Linguística & 8 & $17 \%$ \\
\hline Dados comportamentais & 9 & $17 \%$ \\
\hline Sinais fisiológicos & 9 & $20 \%$ \\
\hline Expressões faciais & 21 & $46 \%$ \\
\hline
\end{tabular}

Figura 2: Categorias por tipos de fontes de dados

Para cada categoria descrita acima, foram investigados quais os instrumentos utilizados para detecção das emoções nos STAs. 24 estudos primários utilizaram a câmera para o reconhecimento da emoção por meio da face, sendo analisadas as características das sobrancelhas, olhos e boca (Figura 3). Apesar da pupila ser classificada dentro da mesma categoria de expressões faciais, ela utiliza outros dispositivos (rastreador ocular) para detecção da emoção do restante da face, sendo assim, classificada em outro subtópico. Somente dois estudos primários investigaram o uso de rastreadores oculares para reconhecer a emoção do estudante. É possível observar que o Encefalograma (ECG), o Eletrocardiograma (EEG) e a Resposta Galvânica da pele (GSR) foram as fontes de reconhecimento da emoção menos investigadas, com apenas um estudo de cada. Acredita-se que a escassez de estudos, investigando essas fontes de dados, seja devido aos altos custos dos aparelhos. Alguns instrumentos ainda não são investigados nos STAs, tais como o reconhecimento da emoção do estudante utilizando instrumentos como a medição do nível de oxigênio no sangue e pressão do mouse (Wu et al., 2015). Outros instrumentos como fotopletismografia, observação e interações face a face também são utilizados na computação afetiva em outros domínios de aplicação que não a Educação, entretanto ainda não investigados nos STAs.

Vale destacar que a fonte de dados para o reconhecimento da emoção, por meio da voz, é também pouco investigada, entretanto, pode-se utilizar dispositivos baratos para sua detecção. Uma possível razão seria a pouca existência de STAs com comunicação via voz com o estudante. 
O principal desafio de sua implementação é conseguir algoritmos capazes de analisar tanto os aspectos verbais (e.g. semântica das palavras) quanto os não verbais (e.g. entonação ou volume).

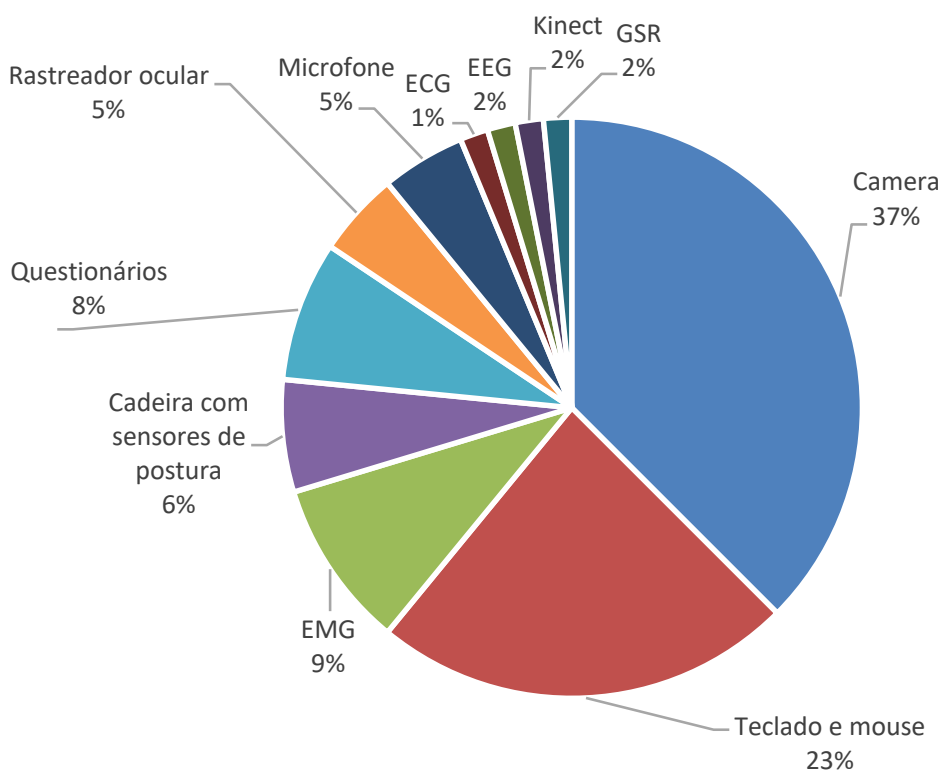

Figura 3 - Estudos primários classificados de acordo com o tipo de instrumento usado para detecção

A Figura 4 e a Tabela 1 apresentam detelhadamente os principais instrumentos utilizados nos STAs para o reconhecimento da emoção do estudante distribuídos por ano. A câmera é o instrumento mais utilizado, tendo o seu surgimento em 2006. Este dispositivo apresenta bom custo-benefício e há variedades de algoritmos disponíveis para o reconhecimendo da emoção, por meio da expressão facial. Outros dispositivos de reconhecimento barato e de fácil acesso são o teclado e mouse, os quais geram um log que possibilita a análise dos dados comportamentais dos estudantes. Dentre os sinais fisiológicos, a eletromiografia e cadeiras com sensores de postura são consideradas as mais populares.

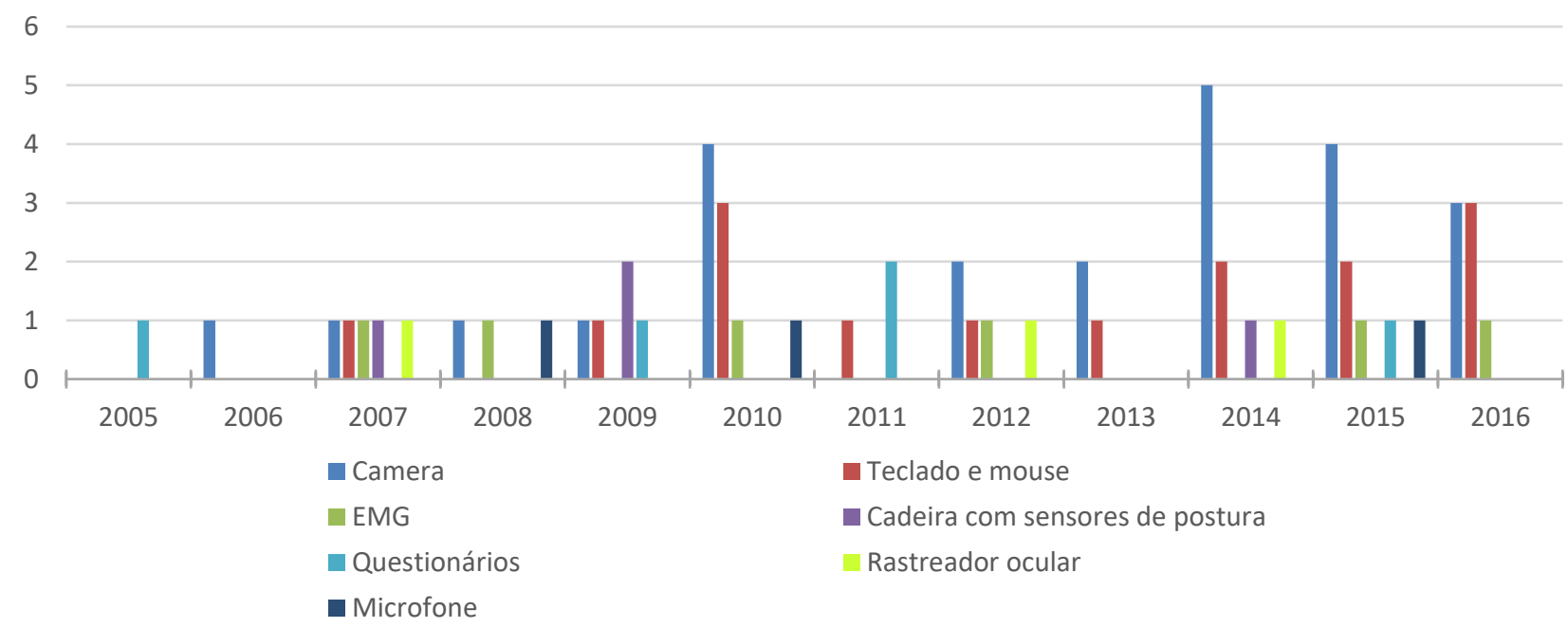

Figura 4 - Sete principais instrumentos utilizados para o reconhecimento emocional nos STAs

Tabela 1 - Instrumentos utilizados para o reconhecimento emocional nos STAs por ano mundialmente

\begin{tabular}{|l|c|c|c|c|c|c|c|}
\cline { 2 - 7 } \multicolumn{1}{c|}{} & \multicolumn{9}{c|}{ Ano } \\
\hline Instrumentos & $2005-2008$ & $\mathbf{2 0 0 9 - 2 0 1 1}$ & $\mathbf{2 0 1 2 - 2 0 1 3}$ & $\mathbf{2 0 1 4}$ & $\mathbf{2 0 1 5}$ & $\mathbf{2 0 1 6}$ & Total \\
\hline
\end{tabular}




\begin{tabular}{|l|r|r|r|r|r|r|r|} 
Câmera & 3 & 5 & 4 & 5 & 4 & 3 & 24 \\
\hline Teclado e mouse & 1 & 5 & 2 & 2 & 2 & 3 & 15 \\
\hline EMG & 2 & 1 & 1 & 0 & 1 & 1 & 6 \\
\hline Cadeira com sensores de postura & 1 & 2 & 0 & 1 & 0 & 0 & 4 \\
\hline Questionários & 1 & 3 & 1 & 0 & 1 & 0 & 5 \\
\hline Rastreador ocular & 1 & 0 & 1 & 1 & 0 & 0 & 3 \\
\hline Microfone & 1 & 1 & 0 & 0 & 1 & 0 & 3 \\
\hline ECG & 0 & 0 & 1 & 0 & 0 & 0 & 1 \\
\hline EEG & 0 & 0 & 1 & 0 & 0 & 0 & 1 \\
\hline Kinect & 0 & 0 & 1 & 0 & 0 & 0 & 1 \\
\hline GSR & 0 & 0 & 1 & 0 & 0 & 0 & 1 \\
\hline
\end{tabular}

Um comparativo com os instrumentos utilizados para reconhecer a emoção do aluno nacionalmente (Tabela 2) expõe que somente foram utilizados o teclado e mouse (por meio de $\log$ ) e questionários de autorrelato. Este último não é considerado muito confiável, pois os estudantes podem dar falsos relatos sobre a sua emoção durante o processo de ensino aprendizagem (Muñoz et al., 2011b). O restante dos instrumentos utilizados para o reconhecimento nos STAs, investigados internacionalmente, apesar de serem de baixo custo e facilmente empregados, ainda não foram utilizados, como é o caso da câmera para o reconhecimento de expressões faciais.

\begin{tabular}{l} 
Tabela 2 - Instrumentos utilizados para o reconhecimento emocional nos STA \\
\cline { 2 - 4 } \multicolumn{4}{|c|}{ Ano } \\
$\qquad$\begin{tabular}{|lr|r|r|}
\hline Instrumentos & $\mathbf{2 0 0 5}$ & $\mathbf{2 0 1 2}$ & $\mathbf{2 0 1 4}$ \\
\hline Teclado e mouse & 1 & 2 & 1 \\
\hline Questionários & 2 & 0 & 0 \\
\hline
\end{tabular}
\end{tabular}

A Figura 5 apresenta os estudos primários agrupados por data de publicação nas bibliotecas digitais em inglês. Como pode ser observado na Figura 5, há um aumento recente da investigação de reconhecimento das emoções do estudante nos STAs. As primeiras publicações surgiram em 2006, provavelmente pelo surgimento e aprimoramento das técnicas de reconhecimento emocional por meio de dispositivos computacionais. Desde esse período, todos os anos são publicados artigos sobre o tema, revelando que há interesse na área para pesquisas.

No Brasil, a primeira publicação ocorreu em 2005, com um estudo primário. Depois, dois estudos primários foram publicados em 2012 e um estudo primário em 2014. Ao comparar com os estudos primários retornados das bases digitais internacionais, é possível observar que há uma carência de pesquisa na área nacionalmente, indicando que é um novo tópico de pesquisa com espaço para novas descobertas e melhorias. 


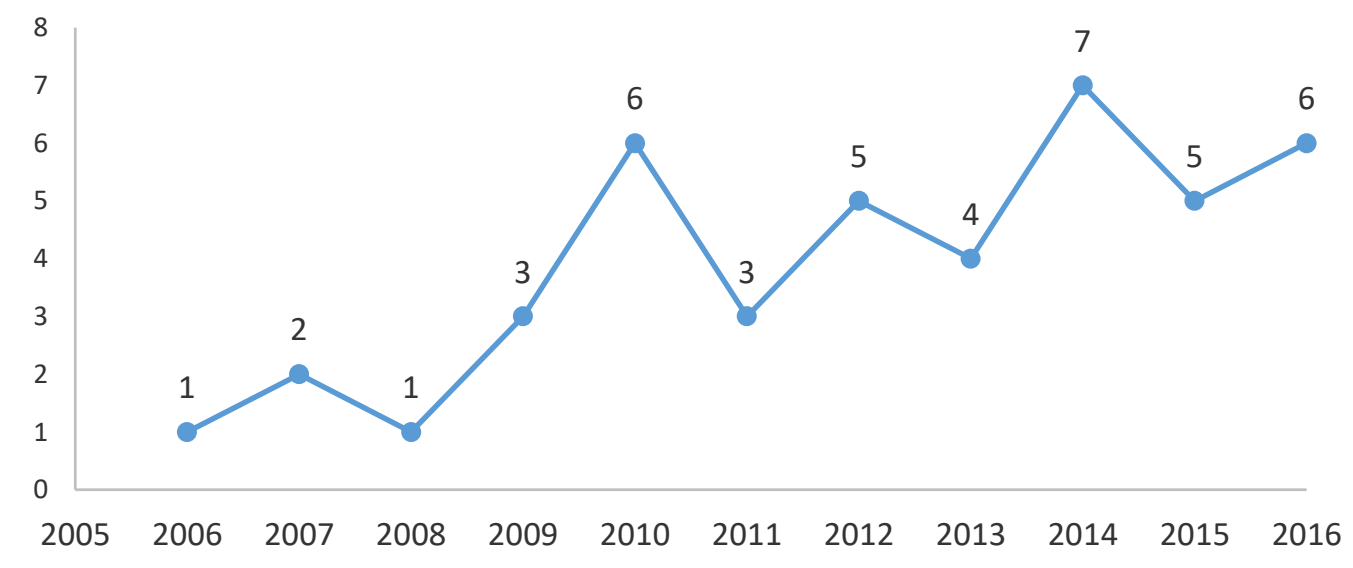

Figura 5: Distribuição dos estudos primários por ano em bibliotecas digitais internacionais

Respondendo a QP3 (Quais são os principais tipos de STAs desenvolvidos?), por meio da Tabela 3, é possível identificar quais são os principais tipos de STAs. Os STAs que possuem interfaces textuais (que as informações são apresentadas somente em forma de texto), com 73\% de todos os estudos primários, são o tipo de STAs mais investigado. Uma das possíveis justificativas para esse resultado são que as interfaces textuais são menos custosas em serem desenvolvidas, envolvendo menos profissionais de outras áreas, como designers, animadores e sonoplastas. Porém, estas não conseguem oferecer o mesmo grau de interação que os STAs que possuem interfaces Serious Games $^{1}$ (representando 18\% dos estudos primários). As interfaces Serious Games oferecem uma classe de jogos capazes de simular situações práticas do dia-a-dia, com objetivo de proporcionar treinamento para jovens, adultos, crianças ou até mesmo para profissionais em situações críticas. Como as interfaces Serious Games conseguem simular ambientes mais realistas do que interfaces textuais, é possível que as emoções sentidas pelos estudantes durante o treinamento em Serious Games sejam mais fiéis à realidade. Do total de oito estudos primários classificados na categoria Serious Games, quatro correspondem a estudos publicados no Brasil.

Tabela 3 - Estudos primários categorizados de acordo com tipo de sistema

\begin{tabular}{|c|c|c|}
\hline Tipo & Frequência & Porcentagem (\%) \\
\hline Textual & 32 & $73 \%$ \\
\hline Serious Games & 8 & $18 \%$ \\
\hline Não especificado & 4 & $9 \%$ \\
\hline
\end{tabular}

Em relação aos tipos de publicação, foram analisados os estudos primários publicados em conferências, periódicos, e como capítulos de livros (Figura 6). A maioria dos estudos selecionados (22 estudos) foi publicada em periódicos, em segundo lugar em conferência (11 trabalhos), e em terceiro como capítulo de livro (sete estudos). A Figura 6 apresenta a quantidade de estudos primários de acordo com o tipo de publicação. É importante mencionar que as áreas de interesse dos estudos selecionados, em sua maioria, apresentam forte relação com diversas subáreas da ciência da computação e com educação.

\footnotetext{
${ }^{1}$ São jogos com objetivo de transmitir conteúdo educacional ou de treinamento (Medonça e Mustaro, 2011).
} 


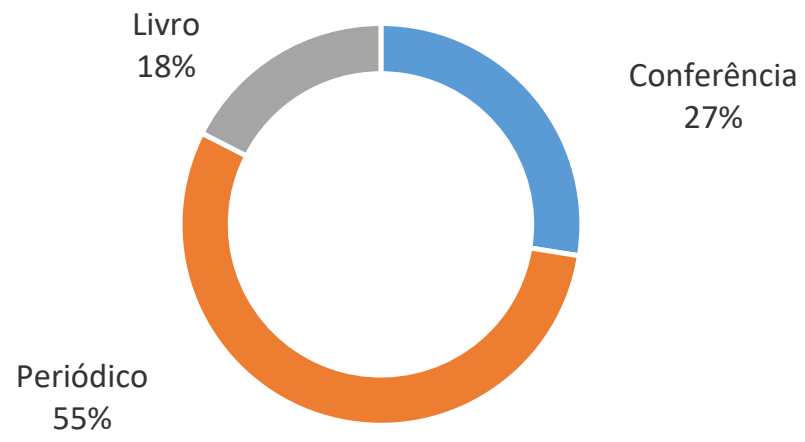

\begin{tabular}{|c|c|c|}
\hline Tipo & Frequência & Porcentagem (\%) \\
\hline Periódico & 22 & $55 \%$ \\
\hline Conferência & 11 & $27 \%$ \\
\hline Capítulo de livro & 7 & $18 \%$ \\
\hline
\end{tabular}

Figura 6 - Estudos primários categorizados de acordo com tipo de publicação

A Tabela 4 apresenta a quantidade de estudos primários publicados por país. É possível observar que o Estados Unidos da América (EUA) é o país com maior número de publicações que relacionam o reconhecimento da emoção do estudante dentro dos STAs (32\% dos estudos primários), seguido do México, com $18 \%$ dos estudos primários. Brasil possui cinco publicações, sendo quatro em território nacional e uma publicação internacional. Países como China, Filipinas, Índia, Irã, Austrália e Tunísia possuem somente uma publicação cada.

Tabela 4 - Estudos primários publicados por país

\begin{tabular}{|l|c|c|}
\hline País & Quantidade & Porcentagem (\%) \\
\hline EUA & 16 & $32 \%$ \\
\hline México & 9 & $18 \%$ \\
\hline Brasil & 5 & $10 \%$ \\
\hline Canadá & 4 & $8 \%$ \\
\hline Taiwan & 4 & $4 \%$ \\
\hline França & 2 & $4 \%$ \\
\hline UK & 2 & $4 \%$ \\
\hline $\begin{array}{l}\text { Nova } \\
\text { Zelândia }\end{array}$ & 2 & $2 \%$ \\
\hline China & 1 & $2 \%$ \\
\hline Filipinas & 1 & $2 \%$ \\
\hline India & 1 & $2 \%$ \\
\hline Irã & 1 & $2 \%$ \\
\hline Tunísia & 1 & $2 \%$ \\
\hline Austrália & 1 & \\
\hline
\end{tabular}

Para analisar além das estatísticas básicas e aprofundar nos resultados (Kaye, 2009), foi avaliado o número médio de autores por artigo. Esta análise utilizou o teste de Mann-Kendall nãoparamétrico para avaliar se há uma tendência significativa, considerando $\alpha=0,05$. Os resultados 
mostram um aumento significativo da tendência de número médio de autores por artigo $(\tau=0,630$; $p=0,006)$. Isto indica que há mais colaboração entre os autores e menos artigos estão sendo escritos por apenas um ou dois autores. Isso pode ser observado na Figura 7, que detalha as tendências do número de autores por artigo.

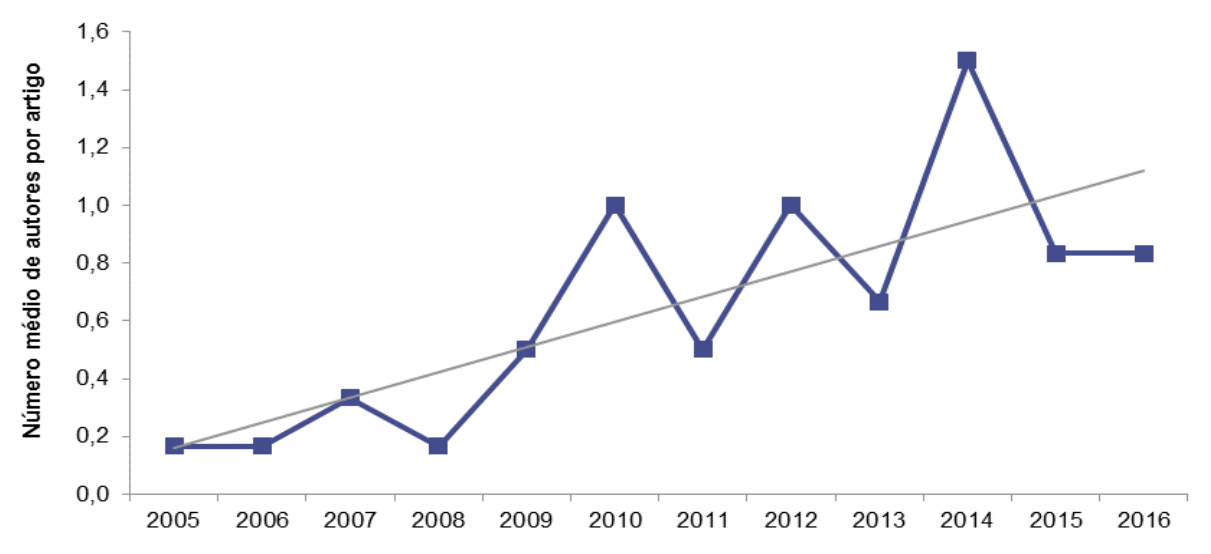

Figura 7 - Tendências do número de autores por artigo

Dos 40 estudos primários retornados, somente quatro não descreveram nenhum experimento empírico. Seis estudos primários realizaram experimentos com especialistas da área, por meio de questionários, guia de diretrizes e testes de mesa. Os 30 estudos primários restantes realizaram experimentos empíricos com estudantes reais de diversas escolaridades, sendo que no final da maioria dos experimentos foram aplicados questionários para verificar se a emoção reconhecida pelo STA era de acordo com a emoção do aluno no momento do processo de ensinoaprendizagem.

A Tabela 5 apresenta a escolaridade dos participantes dos experimentos. A maioria dos participantes foram estudantes do ensino superior (com 39\%), ensino médio (com 14\%) e especialistas (com 11\%). Somente um estudo primário foi categorizado como ensino profissional, caracterizando-se como treinamento de militares em situações interculturais e negociações com outras nacionalidades. A categoria "Outros" refere-se a pessoas com diversas idades, sem estarem frequentando algum curso específico. Dois estudos primários do Brasil realizaram experimentos empíricos com estudantes do ensino primário.

Tabela 5 - Escolaridade dos participantes dos experimentos empíric
\begin{tabular}{|l|c|c|}
\hline \multicolumn{1}{|c|}{ Escolaridade } & Quantidade & Frequência \\
\hline Ensino superior & 17 & $39 \%$ \\
\hline Ensino médio & 6 & $14 \%$ \\
\hline Especialistas & 5 & $11 \%$ \\
\hline Ensino fundamental & 4 & $9 \%$ \\
\hline Não especificados & 4 & $9 \%$ \\
\hline Ensino primário & 4 & $9 \%$ \\
\hline Outros & 3 & $7 \%$ \\
\hline Ensino profissional & 1 & $2 \%$ \\
\hline
\end{tabular}


A Tabela6 apresenta as oito emoções mais investigadas dentro dos STAs, respondendo a QP2 (Quais são as principais emoções detectadas nos STAs?). A maioria dos estudos primários investigaram o reconhecimento de cinco emoções básicas (i.e. raiva, surpresa, aversão, medo e tristeza), entretanto, a emoção mais investigada é o tédio (com 17 estudos primários) e que compõe a categoria de emoções secundárias. Outras emoções secundárias investigadas foram confusão e frustração, com 15 estudos primários cada.

Tabela 6 - Emoções mais investigadas dentro dos STAs

\begin{tabular}{|l|c|c|}
\hline \multicolumn{1}{|c|}{ Emoção } & Quantidade & Categoria \\
\hline Raiva & 13 & \multirow{2}{*}{ Básica } \\
\cline { 1 - 2 } Surpresa & 12 & \\
\cline { 1 - 2 } Aversão & 11 & \multirow{2}{*}{ Secundária } \\
\cline { 1 - 2 } Medo & 11 & \\
\hline Tristeza & 11 & \\
\hline Tédio & 17 & \\
\cline { 1 - 2 } Confusão & 15 & \\
\hline Frustração & 15 & \\
\hline
\end{tabular}

Em relação aos STAs que possuem como interface Serious Games (Tabela 7), as emoções tédio, frustração e confusão foram as mais investigadas, com 3 estudos primários. Outras emoções como curiosidade, prazer e engajamento também foram investigadas. É possível observar que a maioria das emoções (i.e. frustração, confusão, curiosidade, prazer) faz parte da categoria de emoções secundárias.

Os dados apresentados na Tabela 6 sugerem que a maior parte dos estudos investigam as emoções básicas (e.g. raiva, medo, felicidade). Esse resultado corrobora com aquele encontrado por D'Mello e Calvo (2013). Entretanto, como apontado por esses autores, essas emoções, na realidade, ocorrem pouco nos ambientes de aprendizagem (ver Desafio 6, na Seção 6). As emoções secundárias (e.g. frustração, confusão, prazer) são mais investigadas nos STAs que possuem interfaces Serious Games.

Tabela 7 - Emoções mais investigadas dentro dos STAs que possuem interface Serious Games

\begin{tabular}{|l|c|l|}
\hline \multicolumn{1}{|c|}{ Emoção } & Quantidade & \multirow{2}{*}{ Categoria } \\
\hline Tédio & 3 & Primária \\
\hline Frustração & 3 & \multirow{3}{*}{ Secundária } \\
\cline { 1 - 2 } Confusão & 3 & \\
\cline { 1 - 2 } Curiosidade & 2 & Terciária \\
\hline Prazer & 2 & \\
\hline Engajado & 2 & \\
\hline
\end{tabular}

\section{Discussão}

O propósito deste artigo é apresentar um mapeamento sistemático de estudos primários que investigam STAs com mecanismos de reconhecimento da emoção do estudante, resultando 
descobertas e direcionamento de pesquisas futuras. Os resultados sugerem que as pesquisas têm focado em uma ou mais das seguintes fontes de reconhecimento da emoção do estudante: linguística, expressões faciais, sinais fisiológicos e dados comportamentais (Tabela 8).

Tabela 8: Sumário das principais descobertas relacionadas a fonte de dados do reconhecimento emocional

\begin{tabular}{|c|c|c|}
\hline Tópico & Subtópico & Principal descoberta \\
\hline \multirow[t]{3}{*}{ Linguística } & Voz & $\begin{array}{l}\text { Reconhecimento de vocalizações não faladas, } \\
\text { como entonação, frequência e volume da voz } \\
\text { devem ser consideradas, além da respiração, } \\
\text { gestos corporais, gritos e soluços. }\end{array}$ \\
\hline & Semântica & Reconhecimento do significado das palavras \\
\hline & Questionário & $\begin{array}{l}\text { Reconhecimento dos resultados de questões } \\
\text { diretas }\end{array}$ \\
\hline \multirow[t]{3}{*}{ Expressão facial } & Face & $\begin{array}{l}\text { Reconhecimento das características } \\
\text { geométricas (e.g. posições e as distâncias entre } \\
\text { pontos faciais ou a velocidade de separação } \\
\text { destes) da boca, sobrancelhas ou olhos. }\end{array}$ \\
\hline & & $\begin{array}{l}\text { Reconhecimento das características de } \\
\text { aparência (e.g. surgimento de rugas ou marcas de } \\
\text { expressão) que podem ser observadas pela textura } \\
\text { da pele. }\end{array}$ \\
\hline & Pupila & $\begin{array}{l}\text { Reconhecimento de mudanças de diâmetro e } \\
\text { direção da pupila. }\end{array}$ \\
\hline \multirow[t]{4}{*}{ Sinais fisiológicos } & Eletromiografia & $\begin{array}{l}\text { Reconhecimento de correntes elétricas geradas } \\
\text { por um músculo ou pele. }\end{array}$ \\
\hline & Eletrocardiograma & $\begin{array}{l}\text { Reconhecimento da atividade elétrica do } \\
\text { coração, como verificar a frequência cardíaca } \\
\text { o ritmo. }\end{array}$ \\
\hline & Eletroencefalograma & $\begin{array}{l}\text { Reconhecimento da atividade elétrica do } \\
\text { cérebro }\end{array}$ \\
\hline & Postura & $\begin{array}{l}\text { Reconhecimento da postura por meio de } \\
\text { cadeiras com sensores de postura. }\end{array}$ \\
\hline Dados comportamentais & $\log$ & $\begin{array}{l}\text { Reconhecimento do comportamento do } \\
\text { estudante no sistema, como tempo para realizar } \\
\text { um exercício, pedido de ajuda ou negação de uma } \\
\text { ajuda, velocidade da digitação ou velocidade que } \\
\text { movimenta o mouse. }\end{array}$ \\
\hline
\end{tabular}

O reconhecimento emocional por meio da voz utiliza elementos de vocalizações não faladas, como a entonação, frequência ou volume da voz para determinar a emoção. Além disso, a respiração, gestos corporais e soluços também podem contribuir para o reconhecimento (Sharma e Kim, 2002; Kyrillos et al., 2003; Razak et al., 2005; 86; Tóth et al., 2008).

As emoções reconhecidas por meio das expressões faciais podem utilizar dois mecanismos: reconhecimento por meio das características geométricas e por meio das características de aparência. Nas características geométricas são consideradas as posições e as distâncias entre os pontos faciais ou a velocidade de separação entre eles, em elementos faciais como boca, sobrancelhas e olhos. Nas características de aparência é observado o surgimento de rugas ou marcas de expressão na textura da pele (D'Mello et al., 2010; Rosemberg e Elkman, 1994; Ruch, 1995; Sarrafzadeh et al., 2011). 
Há vários sensores fisiológicos disponíveis que auxiliam no reconhecimento da emoção. O reconhecimento pode ser feito pela análise das conduções elétricas na pele, no cérebro ou coração. No último, é possível verificar a frequência cardíaca ou o ritmo do coração. A inclinação das costas ao verificar a postura também pode ser considerada (Picard, 1997; Allanson e Fairclough, 2004; Lin et al., 2016; Shanabrook et al., 2016).

E, por fim, a análise dos dados comportamentais que o estudante realiza no sistema engloba ações como tempo para realizar um exercício, velocidade de movimentação do mouse na tela ou velocidade da digitação, pedido de ajuda, se realizou o exercício com sucesso ou apresentou erros (Jaques e Vicari, 2007; Lagud e Rodrigo, 2010; Doddannara et al., 2013).

Apesar desses mecanismos de reconhecimento da emoção do estudante estarem disponíveis, esse mapeamento também identificou as limitações sobre o uso deles. Os dispositivos classificados na categoria de sensores fisiológicos, como cadeira para detectar postura, encefalograma, eletromiografia e eletrocardiograma, são equipamentos de alto custo e são complexos de serem manuseados. Outras categorias, como semântica, expressões faciais e dados comportamentais, podem utilizar os dispositivos do próprio computador, como a câmera, o microfone e o mouse. Contudo, para as expressões faciais, recomenda-se câmeras de alta precisão e detalhamento.

Em relação às emoções mais investigadas, foi identificado que cinco emoções básicas como raiva, surpresa, aversão, medo e tristeza são mais estudadas dentro dos STAs. Em contrapartida, STAs que possuem interfaces Serious Games investigam mais emoções secundárias, como confusão e frustração.

\section{Ameaças à validade}

As questões de pesquisa e os critérios de inclusão e exclusão foram definidos no início do mapeamento, com objetivo de garantir uma seleção imparcial. Porém, não pode ser descartada a ameaça em relação à avaliação da qualidade dos estudos incluídos, em que foram selecionados sem qualquer atribuição de pontuação.

Outra possível ameaça identificada é a possibilidade de alguns artigos relevantes não serem incluídos, devido à utilização de um conjunto limitado de bibliotecas digitais. Além disso, o sistema de classificação e categorias elaborados representam também uma ameaça à validade. Como mostrado por Pretorius e Budgen (2008), a melhor maneira de classificar e categorizar os resultados somente é obtida ao final da seleção. Além disso, o agrupamento dos estudos em mais de uma categoria pode representar uma ameaça potencial para contagem de frequência e as estatísticas deste estudo de mapeamento.

\section{Conclusão}

Em geral, descobertas anteriores têm mostrado que as emoções aceleram ou perturbam a aprendizagem (Piaget, 1989). Os eventos negativos vivenciados pelos estudantes podem impedir a aprendizagem, ativando a ansiedade, a raiva, o tédio e falta de esperança. As emoções positivas podem promover o aprendizado, ativando emoções como engajamento, curiosidade e orgulho (Pekrun, 2011).

O conhecimento dessas descobertas sobre emoções e sua relação com a aprendizagem pode ser incorporado em ambientes educacionais computacionais, por exemplo, os STAs, tendo o objetivo de auxiliar as estratégias instrucionais para maximizar a aprendizagem. Esses sistemas podem responder adequadamente quando a emoção do estudante alterar durante a aprendizagem. 
Por exemplo, um STA pode utilizar vídeos educacionais quando os estudantes apresentam emoções negativas, como tédio e falta de motivação (D'Mello et al., 2010).

Contudo, responder adequadamente a emoção do estudante pode ser complexo. Pouco se sabe a causa de os estudantes vivenciarem diferentes emoções durante sua interação com os sistemas. Afinal, as emoções podem ser vivenciadas devido à complexidade em acessar o material didático ou devido ao estudante interagir pela primeira vez com o sistema (Harley et al., 2016). Além disso, são necessárias investigações mais profundas para saber quais emoções são relevantes para a aprendizagem, bem como quais caminhos devem ser seguidos utilizando essas emoções para fazer o ensino e a aprendizagem mais efetivos. Do ponto de vista de modelos e métodos para reconhecer a emoção, há um grande avanço nas pesquisas; mas do ponto de vista de explicar a relação entre emoções e aprendizado, há uma carência de investigações (Hernández et al., 2015).

Muitos dos estudos primários focam no reconhecimento da emoção do estudante, no entanto, como o conteúdo ou a interface podem ser adaptados a partir dos dados afetivos reconhecidos é complexo e tem sido pouco investigado. Além disso, há um aumento de interesse em pesquisas que utilizam os agentes pedagógicos para acompanhar o aprendizado do estudante e engajá-lo.

Uma outra oportunidade de pesquisa é a regulação do estudante durante o processo de ensino-aprendizagem. É necessário identificar quais emoções devem ser reguladas, em quais momentos são adequadas e de quais maneiras, ainda não são investigadas. Por exemplo, a pesquisa de Reis et al. (2018) investigou as emoções secundárias durante o aprendizado de álgebra em um software educacional. Os resultados apontaram que estudantes que apresentaram personalidade de neuroticismo sentiam mais rapidamente emoções como confusão e tédio. Esses mesmos resultados foram verificados no estudo de D'Mello e Calvo (2013), em que os estudantes que sentiam confusão poderiam sentir duas outras emoções facilmente: flow e frustração. Caso a emoção não fosse regulada adequadamente ao perfil do estudante (e.g. permitir que sinta com uma determinada duração), essa emoção poderia se transformar em frustração e logo após tédio, que estão negativamente correlacionadas à aprendizagem.

\section{Referências}

Adams, D.M.; McLaren, B.M; Durkin, K. ; Mayer, R.E.; Rittle-Johnson, B.; Isotani, S; Velsen, M.V. (2014). Using erroneous examples to improve mathematics learning with a web-based tutoring system Computers in Human Behavior, 36 (2014), pp. 401-411. doi: 10.1016/j.chb.2014.03.053.

Allanson, J., Fairclough, S.H. (2004). A research agenda for physiological computing. Interacting with Computers 16 (5), 857-878. doi: 10.1016/j.intcom.2004.08.001.

Allen, L. K. ; Mills, C.; Jacovina, M. E.; Crossley, S.; D'Mello, S. e McNamara, D. S. (2016).Investigating Boredom and Engagement during Writing Using Multiple Sources of Information: The Essay, The Writer, and Keystrokes. In Proceedings of the Sixth International Conference on Learning Analytics \& Knowledge (LAK '16). ACM, New York, NY, USA, 114-123. doi: 10.1145/2883851.2883939.

Alzoubi, O.; D'Mello, S. e Calvo, R. (2012). Detecting naturalistic expressions of nonbasic affect using physiological signals. IEEE Transactions on Affective Computing, 3(3): 298-310. doi: 10.1109/T-AFFC.2012.4.

Ammar, M. B.; Neji, M.; Alimi, A. M. e Gouardères, G. (2010). The Affective Tutoring System.Expert Systems with Applications, 37(4): 3013-3023. doi: 10.1016/j.eswa.2009.09.031. 
Arroyo, I.; Cooper, D. G.; Burleson, W.; Woolf, B.P.; Muldner, K. e Christopherson, R. (2009). Emotion Sensors Go To School. In Proceedings of the 2009 conference on Artificial Intelligence in Education: Building Learning Systems that Care: From Knowledge Representation to Affective Modelling, Vania Dimitrova, Riichiro Mizoguchi, Benedict du Boulay, and Art Graesser (Eds.). IOS Press, Amsterdam, The Netherlands, The Netherlands, 17-24. [GS Search]

Azevedo, B.F.T.; Tavares, O.L. e Cury, D. (1999). MUTANTIS - Um Sistema Tutor Inteligente Multi-Agente para o Ensino-Aprendizagem de Conceitos. In: X Simpósio Brasileiro de Informática na Educação, 1999, Curitiba. Anais do X Simpósio Brasileiro de Informática na Educação. Curitiba: Sociedade Brasileira de Computação, 1999. p. 161-168. [GS Search]

Babad, E. (2005). Chapter 7: Nonverbal Behavior in Education. New Handbook of Methods in Nonverbal Behavior Research. Editores: Harrigan, J. e Rosenthal, R. [GS Search]

Baker, R. S.; D'Mello, S. K.; Rodrigo, M. T. e Graesser, A. C. (2010). Better to be frustrated than bored: The incidence, persistence, and impact of learners' cognitive-affective states during interactions with three different computer-based learning environments. International Journal of Human-Computer Studies, 68 (4), 223-241. doi: 10.1016/j.ijhcs.2009.12.003.

Baker, R. S.; Ocumpaugh, J.; Gowda, S. M.; Kamarainen; A. M. e Metcalf, S. J. (2014). Extending Log-Based Affect Detection to a Multi-User Virtual Environment for Science, 8538: 290300. doi: 10.1007/978-3-319-08786-3_25.

Barbosa, N.; Cavalcanti, E. S.; Neves, E. A. L.; Chaves, T. A.; Coutinho, F. A.; e Mortimer, E. F. (2009). A expressividade do professor universitário como fator cognitivo no ensinoaprendizagem. Ciências \& Cognição, 14(1): 75-102. Recuperado em 24 de outubro de 2016, de $\quad$ http://pepsic.bvsalud.org/scielo.php?script=sci arttext\&pid=S180658212009000100006\&lng=pt\&tlng=pt.

Bernacki, M. L., Aleven, V., \& Nokes-Malach, T. J. (2014). Stability and change in adolescents' task-specific achievement goals and implications for learning mathematics with intelligent tutors. Computers in Human behavior, 37, 73-80. doi: 10.1016/j.chb.2014.04.009.

Bicho, L. M. D., \& Pereira, S. R. (2007). Stress ocupacional. Stress Ocupacional. Instituto Politécnico de Coimbra, Departamento de Engenharia Civil, Portugal [periódico na internet].

Borges, S. D. S., Reis, H. M., Durelli, V. H., Bittencourt, I. I., Jaques, P. A., \& Isotani, S. (2013). Gamificação Aplicada à Educação: Um Mapeamento Sistemático. In Anais do Simpósio Brasileiro de Informática na Educação (Vol. 24, No. 1, p. 234). doi: 10.5753/cbie.sbie.2013.234.

Cabada, R.; Estrada, M.; Hernández, F. e Bustillos, R. (2014). Intelligent tutoring system with affective learning for mathematics. Human-Inspired Computing and Its Applications, 8856: 483-493. doi: 10.1007/978-3-319-13647-9 43.

Cabada, R.; Estrada, M.; Hernández, F. e Bustillos, R. (2015). An affective learning environment for Java. IEEE 15th International Conference on Advanced Learning Technologies, Hualien, 2015, pp. 350-354. doi: 10.1109/ICALT.2015.53.

Calvo, R. e D'Mello, S. (2012). Frontiers of affect-aware learning technologies. in IEEE Intelligent Systems, 27(6):86-89. doi: 10.1109/MIS.2012.110.

Carrasco, M.C.O. (2001). Fonoaudiologia Empresarial. São Paulo: Editora Lovise.

Conati, C. (2002). Probabilistic assessment of user's emotions in educational games. Applied Artificial Intelligence, 16(7-8): 555-575. doi: 10.1080/08839510290030390. 
Conati, C.; Maclaren, H. (2009). Empirically building and evaluating a probabilistic model of user affect. User Model. User-Adapted Interact. 19:267-303. doi: 10.1007/s11257-009-9062-8.

Curilem, S.G.; Barbosa; A.R., de Azevedo, F.M. (2007). Intelligent tutoring systems: Formalization as automata and interface design using neural networks, Computers \& Education, 49(3):545-561. doi: 10.1016/j.compedu.2005.10.005.

D'Mello, S. e Calvo, R. (2013). Beyond the basic emotions: what should affective computing compute?. In CHI '13 Extended Abstracts on Human Factors in Computing Systems (CHI EA '13). ACM, New York, NY, USA, 2287-2294. doi: 10.1145/2468356.2468751.

D'Mello, S. e Graesser, A. (2013). AutoTutor and affective autotutor: Learning by talking with cognitively and emotionally intelligent computers that talk back. ACM Trans. Interact. Intell. Syst. 2, 4, Article 23 (January 2013), 39 pages. doi: 10.1145/2395123.2395128.

D'Mello, S. K., Lehman, B. Pekrun, R., e Graesser, A. C. (2014). Confusion Can be Beneficial For Learning, Learning \& Instruction, 29(1), 153-170. doi: 10.1016/j.learninstruc.2012.05.003.

D'Mello, S.; Olney, A.; Williams, C. e Hays, P. (2012). Gaze tutor: A gaze-reactive intelligent tutoring system. International Journal of Human-Computer Studies, 70 (5):377-398. doi: 10.1016/j.ijhcs.2012.01.004.

D'Mello,S.; Lehman, B.; Sullins, J.; Daigle, R.; Combs, R.; Vogt, K. (2010). A time for emoting: when affect-sensitivity is and isn't effective at promoting deep learning. J. Kay, V. Aleven (Eds.), Proceedings of 10th International Conference on Intelligent Tutoring Systems, Springer, Berlin/Heidelberg , pp. 245-254. doi: 10.1007/978-3-642-13388-6 29.

Doddannara, L.; Gowda, S.; Baker, R.; Gowda, S. e De Carvalho, A. (2013). Exploring the relationships between design, students' affective states, and disengaged behaviors within an its, 7926:31-40. doi: 10.1016/S0164-1212(99)00102-8.

Douglas-Cowie, E.; Cowie, R.; Sneddon, I.; Cox, C.; Lowry, O.; McRorie, M. et al. (2007). The HUMAINE database: addressing the collection and annotation of naturalistic and induced emotional data. In A. R. Paiva, R. Prada \& R. Picard (Eds), Affective computing and intelligent interaction (pp. 488-500). Berlin, Heidelberg: Springer. doi: 10.1007/978-3-54074889-2 43 .

Dybå, T. Dingsøyr, T. e Hanssen, G. K. (2007). Applying Systematic Reviews to Diverse Study Types: An Experience Report. In 1st International Symposium on Empirical Software Engineering and Measurement, pages 225-234. doi: 10.1109/ESEM.2007.21.

Ekman, P. (1973). Cross-cultural studies of facial expression. New York: Academic. [GS Search]

Ekman, P. (2003). Emotions revealed (2nd ed.). New York: Times Books. Institute of International Studues. (2004). face to face the science of reading faces part 1: conversation in history With Paul Ekman). University Of California. Berkeley. Disponível em https://www.youtube.com/watch?v=6uABMfsUFls.

Ekman, P. e Davidson, R.J. (1993). Voluntary smiling changes regional brain activity. Psychological Science, 4, 342-345. [GS Search]

Fabron E. M. G. (2006). A voz como recurso didático: Reconhecimento e julgamento de suas qualidades. Tese de Doutorado. Universidade Estadual Paulista, Marília, SP. [GS Search]

Feldman, J.; Goldwasser, G. P. (2004). Eletrocardiograma: recomendações para a sua interpretação. Revista da SOCERJ, 17(4):251- 256. [GS Search]

Fredrickson, B. L. (1998). What good are positive emotions?. Review of general psychology, 2(3):300. doi: $10.1037 / 1089-2680.2 .3 .300$. 
Goldberg, B.; Brawner, K.W.; Holden, H.K. (2012). Efficacy of Measuring Engagement during Computer-Based Training with Low-Cost Electroencephalogram (EEG) Sensor Outputs. In: Proceedings of the Human Factors and Ergonomics Society Annual Meeting, 56(1):198-202. doi: $\underline{10.1177 / 1071181312561018 .}$.

Graesser, A. C. e D’Mello, S. K. (2014). Emotions in Advanced Learning Technologies. In R. Pekrun \& L. Linnenbrink-Garcia (Eds.), International handbook of emotions in education: (pp. 473-493). Routledge: New York, NY. doi: 10.1007/s40593-016-0126-8.

Grafsgaard, J.F.; Wiggins, J.B.; Boyer, K.E.; Wiebe, E.N. e Lester, J.C. (2013). Automatically Recognizing Facial Indicators of Frustration: A Learning-centric Analysis. In Proceedings of the 2013 Humaine Association Conference on Affective Computing and Intelligent Interaction (ACII '13). IEEE Computer Society, Washington, DC, USA, 159-165. doi: 10.1109/ACII.2013.33.

Guimarães, J. I.; Nicolau, J. C.; Polanczyk, C. A.; Pastore, C. A.; Pinho, J. A.; Bacellar, M. S. C.; Ribeiro, D. G. L.; Darwich, R. N.; Ribeiro, A. L. P.; Dunda, M. M. E.; Germiniani, H.; França, F. F.; Saraiva, L.; Ribeiro, C. M. S.; Ginefra, P. G.; Iseu, F.; Eney, R.; Andres, P.; Anisio, F.; Firmiani; A. C.; Grupi, C.; Pinho, C.; Lima, E. V.; Kaiser, E.; Brito, F. S.; Vinhas, G. L. C; Schwartz, H.; Grindler, J.; Aziz, J. L.; Figueiredo, M.; Molina, M. S.; Martinelli, M.; Tobias, N.; Sanches, P. C. R.; Moffa, P. J.; Attanes Neto, S. e Nishioka, S. (2003). Diretriz de interpretação de eletrocardiograma de repouso. Arquivos Brasileiros de Cardiologia, 80(2): 1-18. doi: $10.1590 / \mathrm{S} 0066-782 \mathrm{X} 2003000800001$.

Harley, J. A. e Picard, R. W. (2005). Detecting stress during real-world driving tasks using physiological sensors. Intelligent Transportation Systems, IEEE Transactions On, 6, 156166. doi: $10.1109 /$ TITS.2005.848368.

Harley, J. M.; Bouchet, F.; Hussain, M. S.; Azevedof, R.; Calvo, R. (2015). A multi-componential analysis of emotions during complex learning with an intelligent multi-agent system. Computers in Human Behavior, 48:615-625. doi: 10.1016/j.chb.2015.02.013.

Harley, J.; Carter, C.; Papaionnou, N.; Bouchet, F.; Landis, R.; Azevedo, R. e Karabachian, L. (2016). Examining the predictive relationship between personality and emotion traits and students' agent-directed emotions: towards emotionally-adaptive agent-based learning environments.User Modeling and User-Adapted Interaction 26(2):177-219. doi: $\underline{10.1007 / \mathrm{s} 11257-016-9169-7 .}$.

Harley, J.M.; Bouchet, F. e Azevedo, R. (2013). Aligning and Comparing Data on Emotions Experienced during Learning with MetaTutor. Lecture Notes in Computer Science. Artificial Intelligence in Education, 7926:61-70. doi: 10.1007/978-3-642-39112-5 7.

Hernández, Y.; Arroyo-Figueroa, G. e Sucar, L. (2015). A model of affect and learning for intelligent tutors. Journal of Universal Computer Science, vol. 21, no. 7 (2015), 912-934. doi: 10.3217/jucs-021-07-0912.

Hyun, K.; Kim, E.H. e Kwak, Y.K. (2005). Improvement of emotion recognition by Bayesian classifier using non-zero-pitch concept. In: IEEE International Workshop on Robot and Human Interactive Communication:312-316. doi: 10.1109/ROMAN.2005.1513797.

Iliou, T. e Anagnostopoulos, C.N. (2009). Comparison of different classifiers for emotion recognition. In: IEEE. 13th Panhellenic Conference on Informatics: 102-106. doi: 10.1109/PCI.2009.7.

Jaques, N.; Conati, C.; Harley, J. \& Azevedo, R. (2014). Predicting affect from gaze data during interaction with an intelligent tutoring system. v8474. pp 29-38. doi: 10.1007/978-3-31907221-0 4 . 
Jaques, P. A.; Nunes, M. A. S. N. (2012). Ambientes Inteligentes de Aprendizagem que inferem, expressam e possuem emoções e personalidade. In: Seiji Isotani e Fernanda C. A. Campos. (Org.). Jornada de Atualização em Informática na Educação- JAIE 2012. 1ed.Porto Alegre: SBC, 2012, v. 1, p. 32-71. [GS Search]

Jaques, P. e Vicari, R. (2007). A BDI approach to infer student's emotions in an intelligent learning environment.Computers \& Education. 49(2): 360-384. doi: 10.1016/j.compedu.2005.09.002.

Jaques, P.A.; Nunes, M.A.S.N., Isotani, S. e Bittencourt, Ig. (2012). Computação Afetiva aplicada à Educação: Dotando Sistemas Tutores Inteligentes de Habilidades Sociais. Workshop de Desafios da Computação Aplicada à Educação (desafie). [GS Search]

Jiménez, S.; Juárez-Ramírez R.; Navarro, R.; Coronel A. e Castillo, V. H. (2016). "Architecting an Intelligent Tutoring System with an Affective Dialogue Module," 2016 4th International Conference in Software Engineering Research and Innovation (CONISOFT), Puebla, 2016, pp. 122-129. doi: 10.1109/CONISOFT.2016.27.

Kapoor, A.; Burleson, W. e Picard, R. W.(2007). Automatic prediction of frustration. Int. J. Human-Computer Studies 65:724-736. doi: 10.1016/j.ijhcs.2007.02.003.

Kaye, J. J. (2009). Some statistical analyses of CHI. In Proceedings of the SIGCHI conference on human factors in computing systems (CHI '2009) — extended abstracts (alt.chi) (pp. 25852593). doi: $10.1145 / 1520340.1520364$.

Khan, R.; A. Meyer, H. Konik, and S. Bouakaz (2011). Facial expression recognition using entropy and brightness features. In 11th International Conference on Intelligent Systems Design and Applications (ISDA). doi: 10.1109/ISDA.2011.6121744.

Khan, R.; A. Meyer, H. Konik, and S. Bouakaz (2013). Framework for reliable, real-time facial expression recognition for low resolution images. Pattern Recognition Letters 34(10), 11591168. doi: 10.1016/j.patrec.2013.03.022.

Koedinger, K. R.; Aleven, V.; Roll, I.; e Baker, R. (2009). In vivo experiments on whether supporting metacognition in intelligent tutoring systems yields robust learning. Handbook of metacognition in education, 897-964. [GS Search]

Koelstra, S.; Muhl, C.; Soleymani, M.; Jong-Seok, L.; Yazdani, A.; Ebrahimi, T. et al (2012). DEAP: a database for emotion analysis; using physiological signals. Affective Computing, IEEE Transactions On, 3, 18-31. doi: 10.1109/T-AFFC.2011.15.

Kyrillos, L.R.; Cotes, C. e Feijó, D. (2003). Voz e corpo na TV: a fonoaudiologia a serviço da comunicação. São Paulo: Editora Globo. [GS Search]

Lagud, M. e Rodrigo, M. (2010). The affective and learning profiles of students using an intelligent tutoring system for Algebra. Intelligent Tutoring Systems, 6094:255-263. doi: 10.1007/978-3-642-13388-6_30.

Lane, H. C. (2006). Intelligent tutoring systems: Prospects for guided practice and efficient learning. In Whitepaper for the Army's Science of Learning Workshop (p. 1). doi: 10.1.1.97.9593.

Latham, A., Crockett, K., \& McLean, D. (2014). An adaptation algorithm for an intelligent natural language tutoring system. Computers \& Education, 71, 97-110. doi: 10.1016/j.compedu.2013.09.014.

Lehman, B., D'Mello, S.; Strain, A.; Mills, C.; Gross, M.; Dobbins, A.; Wallace, P.; Millis, K.; e Graesser, A. (2013). Inducing and Tracking Confusion with Contradictions during Complex Learning. Int. J. Artif. Intell. Ed. 22, 1-2 (January 2013), 85-105. doi: 10.1007/978-3-64221869-9 24. 
Levenson, R. W.; Ekman, P.; Heider, K. e Friesen, W. V. (1992). Emotion and autonomic nervous system activity in the Minangkabau of West Sumatra. Journal of Personality and Social Psychology, 62, 972-288. doi: 10.1037/0022-3514.62.6.972.

Lin, H. C. K., Su, S. H., Chao, C. J., Hsieh, C. Y., \& Tsai, S. C. (2016). Construction of Multimode Affective Learning System: Taking Affective Design as an Example. Educational Technology \& Society, 19 (2), 132-147. [GS Search]

Lin, H.-C.; Chao, C.-J. b. e Huang, T.-C. (2015). From a perspective on foreign language learning anxiety to develop an affective tutoring system. Educational Technology Research and Development, 63(5):727-747. doi: 10.1007/s11423-015-9385-6.

Lin, H-C. K.; Wu, C.-H.; Hsueh, Y.-P. (2014) The influence of using affective tutoring system in accounting remedial instruction on learning performance and usability. Computers in Human Behavior, 41:514-522. doi: 10.1016/j.chb.2014.09.052.

Lin, Y.L. e Wei, G. (2005). Speech emotion recognition based on HMM and SVM. In: IEEE. Proceedings of 2005 International Conference on Machine Learning and Cybernectics, 8:4898-4901. doi: 10.1109/ICMLC.2005.1527805.

Mao, X. e Li, Z. (2010). Agent based affective tutoring systems: A pilot study. Computers \& Education, 55(1):202-208. doi: 10.1016/j.compedu.2010.01.005.

Matsumoto, D., e Hwang, H. S. (2011). Training the ability to read microexpressions of emotion improves emotional competence on the job. Motivation \& Emotion. Motivation and Emotion 35(2):181-191. [GS Search]

McNeill, D. (1995) Hand and Mind: What Gestures Reveal about Thought. Chicago: Univesity of Chicago Press. [GS Search]

Medonça, L.R. e Mustaro, N.P. (2011). Elementos imersivos e de narrativa como fatores motivacionais em serious games In: SBC - Proceedings of SBGames, Salvador, BA, Brasil, 2011. [GS Search]

Mehrabian, A. (1971). Silent messages, Wadsworth, California: Belmont. [GS Search]

Morais, F., da Silva, J., Reis, H., Isotani, S., \& Jaques, P. (2017, October). Computação Afetiva aplicada à Educação: uma revisão sistemática das pesquisas publicadas no Brasil. In Brazilian Symposium on Computers in Education (Simpósio Brasileiro de Informática na EducaçãoSBIE) (Vol. 28, No. 1, p. 163). [GS Search]

Muñoz, K.; Kevitt, P.; Lunney, T.; Noguez, J. \& Neri, L. (2011). PlayPhysics: An emotional games learning environment for teaching physics. Knowledge Science, Engineering and Management, 6291:400-411. doi: 10.1007/978-3-642-15280-1_37.

Muñoz, K.; Mc Kevitt, P. ; Lunney, T.; Noguez, J e Neri, L. (2011)b. An emotional student model for game-play adaptation, Entertainment Computing, 2(2):133-141. doi: 10.1016/j.entcom.2010.12.006.

Nkambou, R. b.(2006). A framework for affective intelligent tutoring systems.2006 7th International Conference on Information Technology Based Higher Education and Training, Ultimo, NSW, 2006, pp. nil2-nil8. doi: 10.1109/ITHET.2006.339720.

Norman, D. A. (1981). Twelve Issues for Cognitive Science. In Perspectives on Cognitive Science (pp. 265-295). Hillsdale, NJ: Erlbaum. doi: 10.1016/S0364-0213(81)80002-X.

Nunes, T.M., Jaques, P.A. (2014). Animated Pedagogical Agents as a non-restrictive approach to Gaming The System. Revista Brasileira de Informática na Educação, 22 (1): 147-163. doi: 10.5753/RBIE.2014.22.01.147. 
Overmyer, S.P. (1999). If only my intelligent pedagogical agent could! Journal of Educational Technology \& Society, 2(1). [GS Search]

Padrón-Rivera, G.; Rebolledo-Mendez, G.; Parra, P. \& Huerta-Pacheco, N. (2016). Identification of Action Units Related to Affective States in a Tutoring System for Mathematics. Educational Technology \& Society 19 (2), 77-86. doi: stable/jeductechsoci.19.2.77.

Pekrun, R. (2006). The control-value theory of achievement emotions: assumptions, corollaries, and implications for educational research and practice. Educ. Psychol. Rev. 18, 315-341. doi: $\underline{10.1007 / \mathrm{s} 10648-006-9029-9 .}$.

Pekrun, R. (2011). Emotions as drivers of learning and cognitive development. In: Calvo, R.A., D’Mello, S.K. (eds.) New Perspectives on Affect and Learning Technologies, pp. 23-39. Springer, New York. doi: 10.1007/978-1-4419-9625-1_3.

Petersen, K., Feldt, R., Mujtaba, S. \& Mattsson, M. (2008) Systematic mapping studies in software engineering. Proceedings of the 12th international conference on Evaluation and Assessment in Software Engineering, 68-77. [GS Search]

Piaget, J. (1989) Les relations entre l'intelligence et l'affectivité dans le developpement de l'enfant. Rimé, B.; Scherer, K. (Eds.) Les Émotions. Textes de base en psychologie . Paris: Delachaux et Niestlé, 1989. pp. 75-95. [GS Search]

Picard, R.W. (1997). Affective Computing. MIT Press, Cambridge, MA. [GS Search]

Picard, R.W. (1998). Towards Agents that Recognize Emotion. In Proceedings of IMAGINA, Monaco. [GS Search]

Pretorius, R. e Budgen, D. (2008) A Mapping Study on Empirical Evidence Related to the Models and Forms used in the UML. In 2nd ACM-IEEE International Symposium on Empirical Software Engineering and Measurement (ESEM), pages 342- 344. ACM. [GS Search]

Rajkumar, N. e Ramalingam, V. (2015). Cognitive intelligent tutoring system based on affective state. Indian Journal of Science and Technology, [S.1.], sep. 2015. ISSN $0974-5645$. Available at: <http://www.indjst.org/index.php/indjst/article/view/80145>. Date accessed: 22 Oct. 2016. doi:10.17485/ijst/2015/v8i24/80145.

Razak, A. A. et al. (2005). Comparison between fuzzy and NN method for speech emotion recognition. IEEE. Third International Conference on Information Technology and Applications, 2005. v.1, p. 297-302. doi: 10.1109/ICITA.2005.101.

Reis, Helena M.; Alvares, D. ; Maillard, P. A. J. ; Isotani, S. (2018). Analysis of permanence time in emotional states: A case study using educational software. In: International Conference on Intelligent Tutoring Systems, Montreal. International Conference on Intelligent Tutoring Systems. New York: Springer, 2018. [GS Search]

Reis, R. C. D., Rodriguez, C. L., Lyra, K. T., Jaques, P. A., Bittencourt, I. I., \& Isotani, S. (2015). State of the Art on Affect for Group Formation in CSCL Environments. Brazilian Journal of Computers in Education, 23(03), 113-130. [GS Search]

Reis, H. M.; Maillard, P. A. J. ; Isotani, S. (2017). Sistemas Tutores Inteligentes que reconhecem o estado emocional do estudante: Um mapeamento sistemático. Research and Innovation in Brazilian Education.. 2ed.Palo Alto: Lemann Center, 2017, v. 1, p. 101-104. [GS Search]

Robison, J.L.; Mcquiggan, S.W. e Lester, J.C. (2009). Modeling Task-Based vs. Affect-based Feedback Behavior in Pedagogical Agents: An Inductive Approach. In Proceedings of the 2009 conference on Artificial Intelligence in Education: Building Learning Systems that Care: From Knowledge Representation to Affective Modelling, Vania Dimitrova, Riichiro 
Mizoguchi, Benedict du Boulay, and Art Graesser (Eds.). IOS Press, Amsterdam, The Netherlands, The Netherlands, 25-32. doi: 10.3233/978-1-60750-028-5-25.

Rodrigues, L. M. L., \& Carvalho, M. (2005). STI-I: Sistemas Tutoriais Inteligentes que integram cognição, emoção e motivação. Brazilian Journal of Computers in Education, 13(1). [GS $\underline{\text { Search }}$

Rosemberg, E. L. e Elkman, P. (1994). Coherence between expressive and experiential systems in emotion. Cognition and Emotion, 8, 201-229. doi: 10.1080/02699939408408938.

Ruch, W. (1995). Will the real relationship between facial expression and affective experience please stand up: The case of exhilaration. Cognition and Emotion, 9, 33-58. doi: $\underline{10.1080 / 02699939508408964 .}$

Santos F.M. e Mortimer, E.F. (2001). Comunicação não-verbal em sala de aula. Revista Brasileira de Pesquisa em Educação em Ciências, 11 (1), 18-30. [GS Search]

Sarrafzadeh, A.; Alexander, S.; Dadgostar, F.; Fan, C. e Bigdeli, A. (2008). "How do you know that I don't understand?" A look at the future of intelligent tutoring systems, Computers in Human Behavior, 24(4):1342-1363. doi: 10.1016/j.chb.2007.07.008.

Sarrafzadeh, A.; Shanbehzadeh, A. e Overmyer, S. (2011). Capítulo 7: E-Learning with Affective Tutoring Systems. Intelligent Tutoring Systems in E-Learning Environments: Design, Implementation and Evaluation. Editores: Stankov, S.; Glavinic, V. e Rosic, Marko. Nova York. pp. 129-139. doi: 10.4018/978-1-61692-008-1.

Scherer, K. R. (2000). Psychological models of emotion. In J. Borod (Ed.). The neuropsychology of emotion (pp. 137-162). Oxford/New York: Oxford University Press. [GS Search]

Schneider, K.; Josephs, I. (1991). The expressive and communicative functions of preschool children's smiles in an achievement situation. Nonverbal Behavior 15. doi: 10.1007/BF01672220.

Schyns, P.; L. Petro, and Smith, M. (2009). Transmission of facial expressions of emotion coevolved with their efficient decoding in the brain: Behavioral and brain evidence. PLoS ONE 4(5), e5625. doi: 10.1371/journal.pone.0005625.

Shanabrook, D. H.; Arroyo, I. e Woolf, B. P. (2016). Using Touch as a Predictor of Effort: What the iPad Can Tell Us about User Affective State. User Modeling, Adaptation, and Personalization, 7379:322-327. [GS Search]

Sharma, R. N. U.; Kim, C. (2002). Emotion recognition in spontaneous emotional utterances from movie sequences. In: WSEAS International Conference on Electronics, Control \& Signal Processing. [GS Search]

Silva, M. E. e Silva, P. S. (2016). Elementos da face do professor no cenário tutorial: implicações na formação de enfermeiros. Revista de Pesquisa: Cuidado é Fundamental; 8(1): 3803-3819. doi: 10.9789/2175-5361.2016.v8i1.3803-3819.

Smilek, D.; Carriere, J.S.A. e Cheyne, J.A. (2010). Out of mind, out of sight: eye blinking as indicator and embodiment of mind wandering. Psychological Science, 21 (6): 786-789. doi: $\underline{10.1177 / 0956797610368063 .}$.

Smith, BL; Brown, BL; Strong, WJ; Rencher, A.C. (1975). Effects of speech rate on personality perception. Language and speech. 18 (2): 145-52. doi: 10.1177/002383097501800203.

Stefanelli, M.C. (1993). Comunicação com o paciente. Teoria de ensino. São Paulo: Robe. [GS $\underline{\text { Search }}]$ 
Tao, J.; Tieniu, T. (2005). Affective Computing: A Review. Affective Computing and Intelligent Interaction. LNCS 3784. Springer. pp. 981-995. doi:10.1007/11573548.

Thurman, L. e Welch, G.F. (2000). Body mind \& voice: Foundations of voice education (revised ed.), Collegeville, Minnesota: The Voice Care Network et al., ISBN 0-87414-123-0. [GS $\underline{\text { Search }] ~}$

Tian, Y. L., Kanade, T., \& Cohn, J. F. (2005). Facial expression analysis. Handbook of face recognition, 247-275. [GS Search]

Todorov, J. C. (2012). Sobre uma definição de comportamento. Perspectivas em análise do comportamento, 3(1), 32-37. [GS Search]

Tóth, S. L.; Sztahó, D.; Vicsi, K. (2008). Speech emotion perception by human and machine. In: Verbal and Nonverbal Features of Human-Human and Human-Machine Interaction. Springer. p. 213-224. doi: 10.1007/978-3-540-70872-8_16.

Vail, A. K.; Grafsgaard, J. F.; Boyer, K. E.; Wiebe, E. N. e Lester, J. C. (2016). Gender Differences in Facial Expressions of Affect During Learning. In Proceedings of the 2016 Conference on User Modeling Adaptation and Personalization (UMAP '16). ACM, New York, NY, USA, 65-73. doi: 10.1145/2930238.2930257.

VanLehn, K. (2006). The Behavior of Tutoring Systems. International Journal of Artificial Intelligence in Education, 16(3):227-265. [GS Search]

VanLehn, K. (2011). The Relative Effectiveness of Human Tutoring, Intelligent Tutoring Systems, and Other Tutoring Systems. Educational Psychologist, 46 (4): 197-221. doi: $\underline{10.1080 / 00461520.2011 .611369}$

Vos, H. J. (1995). Applications of Bayesian decision theory to intelligent tutoring systems. Computers in Human Behavior, 11(1), 149-162. doi: 10.1016/0747-5632(94)00029-H.

Wenger, E. (1987). Artificial Intelligence and Tutoring Systems. Morgan Kaufman. [GS Search]

Williams, C.E.; Stevens, K.N. (1972). Emotions and speech: some acoustical correlates. The Journal of the Acoustical Society of America, 52 (4): 1238-50. doi: 10.1121/1.1913238.

Woolf, B. P. (2010). Building intelligent interactive tutors: Student-centered strategies for revolutionizing e-learning. Morgan Kaufmann. [GS Search]

Woolf, B.; Burleson, W.; Arroyo, I.; Dragon, T.; Cooper, D. e Picard, R. (2009). Affect-aware tutors: recognising and responding to student affect. Int. J. Learning Technology, 4(3/4):129:164. doi: 10.1504/IJLT.2009.028804.

Wu, C.-H.; Huang, Y.-M. e Hwang, J-P. (2015). Review of affective computing in education/learning: Trends and challenges. British Journal of Education Technology, 47(6):1304-1323. doi: 10.1111/bjet.12324.

Zatarain-Cabada, R.; Barrón-Estrada, M. L.; Camacho, J. L. O. e Reyes-García, C. A. (2013). Integrating Learning Styles and Affect with an Intelligent Tutoring System. 2013 12th Mexican International Conference on Artificial Intelligence, Mexico City, 2013, pp. 247-253. doi: 10.1109/MICAI.2013.36.

Zatarain-Cabada, R.; Barrón-Estrada, M. L.; Camacho, J.L.O. e Reyes-García, C.A. (2014). Capítulo: Affective Tutoring System for Android Mobiles. Intelligent Computing Methodologies, 8589:1-10. doi: 10.1007/978-3-319-09339-0_1.

\section{Apêndice: Sumário dos estudos primários}


Tabela 1 - Reconhecimento da emoção do estudante dos estudos primários internacionais

\begin{tabular}{|c|c|c|c|c|}
\hline Estudo & $\begin{array}{ll}\text { Agente } & \text { ou } \\
\text { Estudante? } & \end{array}$ & Tópico & Subtópico & \\
\hline (Allen et al., 2016) & Estudante & $\begin{array}{l}\text { Expressões faciais/Dados } \\
\text { comportamentais }\end{array}$ & Face/Log & \\
\hline (Alzoubi et al., 2012) & Estudante & Sinais fisiológicos & $\begin{array}{l}\text { Eletrocardiograma } \\
\text { Electromiografia }\end{array}$ & e \\
\hline (Ammar et al., 2010) & Agente/Estudante & Expressões faciais & Face & \\
\hline (Baker et al., 2010) & Estudante & Expressões faciais/Linguística & Face/Semântica & \\
\hline (Baker et al., 2014) & Estudante & $\begin{array}{l}\text { Expressões faciais/Sinais } \\
\text { fisiológicos }\end{array}$ & Face/Postura & \\
\hline (Cabada et al., 2015) & Agente/Estudante & Expressões faciais & Face & \\
\hline $\begin{array}{l}\text { (Calvo e D'Mello, } \\
\text { 2012) }\end{array}$ & Agente/Estudante & Expressões faciais/Linguística & Face/Semântica & \\
\hline (Arroyo et al., 2009) & Estudante & $\begin{array}{l}\text { Sinais fisiológicos/Expressões } \\
\text { faciais/ Dados } \\
\text { comportamentais }\end{array}$ & Postura/Face/Log & \\
\hline $\begin{array}{l}\text { (D'Mello e Graesser, } \\
\text { 2013) }\end{array}$ & Estudante & $\begin{array}{l}\text { Expressões faciais/Sinais } \\
\text { fisiológicos }\end{array}$ & Face/Postura & \\
\hline (D'Mello et al., 2012) & Estudante & Expressões faciais & Pupila & \\
\hline $\begin{array}{l}\text { (Doddannara et al., } \\
\text { 2013) }\end{array}$ & Estudante & Dados comportamentais & $\log$ & \\
\hline $\begin{array}{l}\text { (Grafsgaard et al., } \\
\text { 2013) }\end{array}$ & Estudante & Expressões faciais & Face & \\
\hline $\begin{array}{l}\text { (Goldberg et al., } \\
\text { 2012) }\end{array}$ & Estudante & Sinais fisiológicos & Encefalograma & \\
\hline (Harley et al., 2013) & Estudante & Expressões faciais & Face & \\
\hline (Harley et al., 2015) & Estudante & $\begin{array}{l}\text { Sinais fisiológicos/Expressões } \\
\text { faciais }\end{array}$ & Face/Electromiografia & \\
\hline $\begin{array}{l}\text { (Hernández et al., } \\
\text { 2015) }\end{array}$ & Agente/Estudante & Dados comportamentais & $\log$ & \\
\hline $\begin{array}{l}\text { (Jaques e Vicari, } \\
2007)\end{array}$ & Estudante & Dados comportamentais & $\log$ & \\
\hline (Jaques et al., 2014) & Estudante & Expressões faciais & Pupila & \\
\hline (Jiménez et al., 2016) & Estudante & Linguística & Semântica & \\
\hline (Kapoor et al., 2007) & Estudante & $\begin{array}{l}\text { Sinais fisiológicos/Expressões } \\
\text { faciais }\end{array}$ & $\begin{array}{l}\text { Postura } \\
\text { eletromiografia/Face } \\
\text { Pupila }\end{array}$ & $\begin{array}{l}\mathrm{e} \\
\mathrm{e}\end{array}$ \\
\hline $\begin{array}{l}\text { (Lagud e Rodrigo, } \\
\text { 2010) }\end{array}$ & Estudante & Dados comportamentais & $\log$ & \\
\hline (Lin et al., 2014) & Agente/Estudante & Expressões faciais/Linguística & Face/Semântica & \\
\hline (Lin et al., 2015) & Agente/Estudante & Expressões faciais/Linguística & Face/Semântica & \\
\hline (Lin et al., 2016) & Agente/Estudante & $\begin{array}{l}\text { Sinais fisiológicos/Expressões } \\
\text { faciais }\end{array}$ & Face/Electromiografia & \\
\hline (Mao e Li, 2010) & Agente/Estudante & Expressões faciais/Linguística & Face/Voz & \\
\hline (Muñoz et al., 2011) & Agente/Estudante & Dados comportamentais & $\log$ & \\
\hline
\end{tabular}




\begin{tabular}{|c|c|c|c|}
\hline (Muñoz et al., 2011b) & Estudante & Linguística & Questionário \\
\hline (Nkambou, 2006) & Agente/Estudante & Expressões faciais & Face \\
\hline $\begin{array}{l}\text { (Padrón-Rivera et al., } \\
\text { 2016) }\end{array}$ & Estudante & Expressões faciais & Face \\
\hline $\begin{array}{l}\text { (Rajkumar e } \\
\text { Ramalingam, 2015) }\end{array}$ & Agente/Estudante & Expressões faciais/Semântica & Face/Voz \\
\hline (Robison et al., 2009) & Estudante & Linguística & Questionário \\
\hline $\begin{array}{l}\text { (Sarrafzadeh et al., } \\
\text { 2011) }\end{array}$ & Agente/Estudante & $\begin{array}{l}\text { Sinais fisiológicos/Expressões } \\
\text { faciais }\end{array}$ & Face e Electromiografia \\
\hline $\begin{array}{l}\text { (Sarrafzadeh et al., } \\
\text { 2008) }\end{array}$ & Agente/Estudante & $\begin{array}{l}\text { Sinais fisiológicos/Expressões } \\
\text { faciais }\end{array}$ & Face e Electromiografia \\
\hline $\begin{array}{l}\text { (Zatarain-Cabada et } \\
\text { al., 2013) }\end{array}$ & Estudante & Expressões faciais & Face \\
\hline $\begin{array}{l}\text { (Zatarain-Cabada et } \\
\text { al., 2014) }\end{array}$ & Estudante & Expressões faciais & Face \\
\hline
\end{tabular}

Tabela 2 - Reconhecimento da emoção do estudante dos estudos primários nacionais

\begin{tabular}{|c|c|c|c|}
\hline Estudo & $\begin{array}{ll}\text { Agente } & \text { ou } \\
\text { Estudante? } & \end{array}$ & Tópico & Subtópico \\
\hline $\begin{array}{l}\text { (Nunes e Jaques, } \\
\text { 2014) }\end{array}$ & Estudante & Dados comportamentais & $\log$ \\
\hline $\begin{array}{l}\text { (Jaques e Nunes, } \\
\text { 2012) }\end{array}$ & Estudante & Dados comportamentais & $\log$ \\
\hline $\begin{array}{l}\text { (Rodrigues } \\
\text { Carvalho, 2005) }\end{array}$ & Estudante & Dados Comportamentais & Log/Questionários \\
\hline (Jaques et al., 2012) & Estudante & Dados Comportamentais & $\log$ \\
\hline $\begin{array}{ll}\text { (Rodrigues } & \text { e } \\
\text { Carvalho, 2005b) }\end{array}$ & Estudante & Dados Comportamentais & Questionários \\
\hline
\end{tabular}

